\title{
Prion-Like Seeding of Misfolded $\alpha$-Synuclein in the Brains of Dementia with Lewy Body Patients in RT-QUIC
}

\author{
Kazunori Sano $^{1}$ (D) Ryuichiro Atarashi ${ }^{2} \cdot$ Katsuya Satoh $^{3} \cdot$ Daisuke Ishibashi $^{4}$. \\ Takehiro Nakagaki $^{4}$ - Yasushi Iwasaki ${ }^{5}$ Mari Yoshida ${ }^{5} \cdot$ Shigeo Murayama $^{6}$. \\ Kenichi Mishima $^{1} \cdot$ Noriyuki Nishida $^{4}$
}

Received: 29 December 2016/Accepted: 16 May 2017 /Published online: 26 May 2017

(C) The Author(s) 2017. This article is an open access publication

\begin{abstract}
The prion-like seeding of misfolded $\alpha$-synuclein $(\alpha$ Syn) involved in the pathogenesis of Lewy body diseases (LBD) remains poorly understood at the molecular level. Using the real-time quaking-induced conversion (RT-QUIC) seeding assay, we investigated whether brain tissues from cases of dementia with Lewy bodies (DLB), which contain serine 129 (Ser129)-phosphorylated insoluble aggregates of $\alpha$ Syn, can convert Escherichia coli-derived recombinant $\alpha$ Syn (r- $\alpha$ Syn) to fibrils. Diffuse neocortical DLB yielded $50 \%$ seeding dose $\left(\mathrm{SD}_{50}\right)$ values of $10^{7} \sim 10^{10} / \mathrm{g}$ brain. Limbic DLB was estimated to have an $\mathrm{SD}_{50}$ value of $\sim 10^{5} / \mathrm{g}$
\end{abstract}

Electronic supplementary material The online version of this article (doi:10.1007/s12035-017-0624-1) contains supplementary material, which is available to authorized users.

Kazunori Sano

ksano@fukuoka-u.ac.jp

1 Department of Physiology and Pharmacology, Faculty of Pharmaceutical Sciences, Fukuoka University, 8-19-1 Nanakuma, Jonan-ku, Fukuoka 814-0180, Japan

2 Division of Microbiology, Department of Infectious Diseases, Faculty of Medicine, University of Miyazaki, Miyazaki 889-1692, Japan

3 Department of Locomotive Rehabilitation Science, Nagasaki University Graduate School of Biomedical Sciences, Nagasaki 852-8523, Japan

4 Department of Molecular Microbiology and Immunology, Nagasaki University Graduate School of Biomedical Sciences, Nagasaki 852-8523, Japan

5 Department of Neuropathology, Institute for Medical Science of Aging, Aichi Medical University, Aichi 480-1195, Japan

6 Department of Neuropathology, Tokyo Metropolitan Geriatric Hospital and Institute of Gerontology, Tokyo 173-0015, Japan brain. Furthermore, RT-QUIC assay discriminated DLB from other neurological and neurodegenerative disorders. Unexpectedly, the prion-like seeding was reconstructed in reactions seeded with oligomer-like species, but not with insoluble aggregates of $r-\alpha$ Syn, regardless of Ser129 phosphorylation status. Our findings suggest that RT-QUIC using r- $\alpha$ Syn can be applied to detect seeding activity in LBD, and the culprit that causes prion-like seeding may be oligomeric forms of $\alpha$ Syn.

Keywords $\alpha$-synuclein $\cdot$ Real-time quaking-induced conversion (RT-QUIC) · Prion · Dementia with Lewy bodies (DLB)

\section{Introduction}

The accumulation of abundant misfolded proteins in the brain is a defining feature of most neurodegenerative disorders. Lewy body diseases (LBD), such as dementia with Lewy bodies (DLB) and Parkinson's disease (PD), are characterized by the presence of Lewy bodies (LB), which are filamentous cytoplasmic inclusions composed mainly of aggregated $\alpha$ synuclein ( $\alpha$ Syn). Although the pathogenic mechanisms have not been fully elucidated, LBD is thought to occur through the accumulation of LB, i.e., aggregated $\alpha$ Syn, in neurons and neurites.

The bulk of $\alpha$ Syn aggregates within LB are phosphorylated at serine 129 (Ser129), while $\alpha$ Syn in the normal brain undergoes very little phosphorylation. Phosphorylation at Ser129 is the dominant pathological characteristic and may be crucial in LB formation and the pathogenesis of LBD. Ser129 phosphorylation accelerates polymerization of recombinant $\alpha$ Syn (r- $\alpha$ Syn) [1], and overexpression of wild-type (WT) $r-\alpha$ Syn, but not nonphosphorylatable mutant with 
substitution of alanine for Ser129 (S129A), induced significant accumulation of LB-like inclusions in cultured cells [2]. Other groups demonstrated that Ser129 phosphorylation inhibits the fibrillation of $r-\alpha \operatorname{Syn}[3]$, and mutation of S129A increases inclusion formation in a Drosophila model [4]. The study using Drosophila also revealed that the number of inclusion bodies is inversely correlated with toxicity to dopaminergic neurons, indicating that inclusion bodies protect against neuronal toxicity [4]. However, studies in rat models using recombinant adeno-associated virus (rAAV) to overexpress WT and mutant $\alpha$ Syn indicated that nonphosphorylated $\alpha$ Syn is associated with more severe toxicity toward dopaminergic neurons than the phosphorylated form $[5,6]$, and that there are no significant differences in neurotoxicity between both forms [7]. Thus, the significance of Ser129 phosphorylation of $\alpha$ Syn in the pathogenesis of LBD remains poorly understood.

Postmortem studies by Braak and colleagues showed that LB pathologies initially arise in the medulla oblongata and the olfactory bulb and then extend to the midbrain and the limbic areas, with subsequent spread to the neocortical regions in PD [8]. In addition, the pathological stage appears to be closely linked to progression of lesions and clinical manifestations [9]. The spread of LB in the brain suggests that pathological $\alpha$ Syn retains the ability to propagate as prions. Transplant studies provided evidence that LB are present in normal fetal mesencephalic neurons grafted into the striatum of patients with PD, indicating that the pathology could be transmitted from the host brain to grafted healthy neurons $[10,11]$. Moreover, inoculation of pathological brain homogenates from A53T mutant human $\alpha$ Syn transgenic (Tg) mice showing motor clinical abnormalities into A53T Tg mice [12-14] resulted in LB-like aggregations derived from endogenous $\alpha$ Syn and cell-to-cell spread, and the A53T Tg mice showed reduced survival period [12]. A similar prion-like phenomenon was also induced in WT mice inoculated with brain extracts from DLB patients [15] and A53T Tg mice inoculated with brain homogenates from multiple system atrophy (MSA) patients [14]. Furthermore, introduction of synthetic fibrils formed from $r-\alpha$ Syn elicited intercellular transmission of pathological $\alpha$ Syn in primary neurons [16] and WT mice [17], which developed dopamine neuron loss that resulted in motor deficits. These reports suggested that $\alpha$ Syn behaves like a prion.

Previously, we developed an in vitro amplification technique, designated "real-time quaking-induced conversion (RT-QUIC)," for detection of prions in tissue and body fluids [18]. This assay is based on the propensity of prions to act as seeds and replicate in an autocatalytic process that converts recombinant prion protein as a substrate into amyloid fibrils, which retain the prion infectivity and strain properties of the original prion [19]. The present study was performed to investigate whether prion-like conformational conversion of $\mathrm{r}-\alpha$ Syn is induced by brain tissue from patients with DLB using the RT-QUIC assay. More recently, it has been shown that RT-QUIC using $\mathrm{r}-\alpha$ Syn can detect abnormal $\alpha$ Syn in the brain and cerebrospinal fluid (CSF) from DLB and PD [20]. The RT-QUIC assay of CSF from DLB and PD showed 92 and $95 \%$ sensitivity, respectively, with an overall specificity of $100 \%$. The study provides no data regarding the seeding activity and the form of abnormal $\alpha$ Syn. Furthermore, we performed quantitative analysis of abnormal $\alpha$ Syn seeding activity and examined the relationship between prion-like behavior and Ser129 phosphorylation or multimeric status of $\alpha$ Syn.

\section{Methods}

\section{Patients}

DLB brain tissues were obtained at autopsy from seven patients with histopathological confirmation of the clinical diagnosis. Of these subjects, six were classified as having diffuse neocortical DLB type (DN-DLB), and the remaining one case was limbic DLB type (Li-DLB) according to Braak staging. Brain tissues of prion disease were obtained at autopsy from three patients with sporadic CJD (sCJD) and one patient with GSS associated with a Pro to Leu mutation at codon 102 of $P R N P$ (P102L). sCJD subtypes were diagnosed according to the genotype at codon 129 of the PRNP gene and the physicochemical properties of abnormal prion protein $\left(\mathrm{PrP}^{\mathrm{Sc}}\right)$. They included two cases of type 1, codon 129 MM (MM1), and one case of type 2, codon $129 \mathrm{MM}$ (MM2). Brain tissues with $\mathrm{AD}$ were obtained at autopsy from two patients that had received a neuropathological diagnosis of the presence of neurofibrillary tangles and neuritic plaques. The brain specimens were pure forms of $\mathrm{AD}$ with little or no coexisting LBD. Brain tissues from schizophrenia and breast cancer patients without histopathological changes in the brain were used as nondegenerative cases. All samples were taken from the frontal cortex and stored at $-80^{\circ} \mathrm{C}$.

\section{Recombinant Human $\alpha$-Synuclein Expression and Purification}

The DNA sequence encoding N-terminal His-tagged human wild-type $\alpha$ Syn residues $1-140$ was amplified from human cDNA (Cat. No. FCC139B01; Toyobo) with forward primer (5'-ggaattccatatgaaacatcatcatcatcatcaccagatggatgtattcatgaaagg- $\left.3^{\prime}\right)$ and reverse primer $\left(5^{\prime}\right.$-ctagctagctagttaggcttcaggttcgtagtctt-3'). The S129A mutant was amplified with forward primer $\left(5^{\prime}\right.$-ggaattccatatgaaacatcatcatcatcatcaccagatggatgtattcatgaaagg- $\left.3^{\prime}\right)$ and reverse primer (5'-ctagctagctagttaggcttcaggttcgtagtcttgataccettcctcagcaggc-3'). A stop point for dipeptidyl peptidase I (DAPase, Cat. No. 34362; Qiagen) that digests $\mathrm{N}$-terminal His-tags was introduced into the 
protein sequence by inserting a glutamine codon (cag) into the expression construct. The amplified PCR fragment was inserted into NdeI and NheI sites of the pET11a vector (Cat. No. 69436-3; Novagen) and confirmed by sequence analysis. After transforming the plasmids into competent BL21 DE3 E. coli cells (Cat. No. DS250; BioDynamics Laboratory), recombinant protein was expressed at $37{ }^{\circ} \mathrm{C}$ for $16 \mathrm{~h}$ using MagicMedia E. coli Expression Medium (Cat. No. K6815; Invitrogen). The cells were pelleted by centrifugation at $3000 \mathrm{rpm}$ for $15 \mathrm{~min}$ at $4{ }^{\circ} \mathrm{C}$ were suspended in CelLytic B (Cat. No. B7435; Sigma-Aldrich) in the presence of $1 \mathrm{~g} / \mathrm{ml}$ lysozyme (Cat. No. 120-02674; Wako) and $500 \mathrm{U} / \mathrm{mL}$ benzonase nuclease (Cat. No. 70664-3; Novagen). The lysate was centrifuged at $10000 \mathrm{rpm}$ for $30 \mathrm{~min}$ at $4{ }^{\circ} \mathrm{C}$, and the supernatant was incubated with Ni-NTA Superflow resin
(Cat. No. 30430; Qiagen) at room temperature for $30 \mathrm{~min}$ and then loaded onto a gravity flow column (Muromac mini-column; Muromachi Chemical Inc). The protein was eluted with a buffer containing $300 \mathrm{mM} \mathrm{NaCl}, 50 \mathrm{mM}$ Tris$\mathrm{HCl}$ ( $\mathrm{pH} 8.0$ ), and $250 \mathrm{mM}$ imidazole and dialyzed against $10 \mathrm{mM}$ phosphate buffer ( $\mathrm{pH}$ 7.0) in cellulose dialysis tubing (Cat. No. 68035; ThermoFisher Scientific) at $4{ }^{\circ} \mathrm{C}$ overnight. As shown in Fig. 1a, b, removal of the tag from N-terminal His-tagged human $\alpha$-synuclein was performed using the TAGZyme system (Cat. No. 34300; Qiagen). The purified His-r- $\alpha$ Syn contains a glutamine stop point for N-terminal exopeptidase DAPase between the His-tag sequence and the first amino acid of $\alpha$ Syn. The tag was cleaved by $40 \mathrm{mU}$ DAPase in the presence of $2.4 \mathrm{U}$ glutamine cyclotransferase (Qcyclase, Cat. No. 34342; Qiagen) at $4{ }^{\circ} \mathrm{C}$ overnight. The
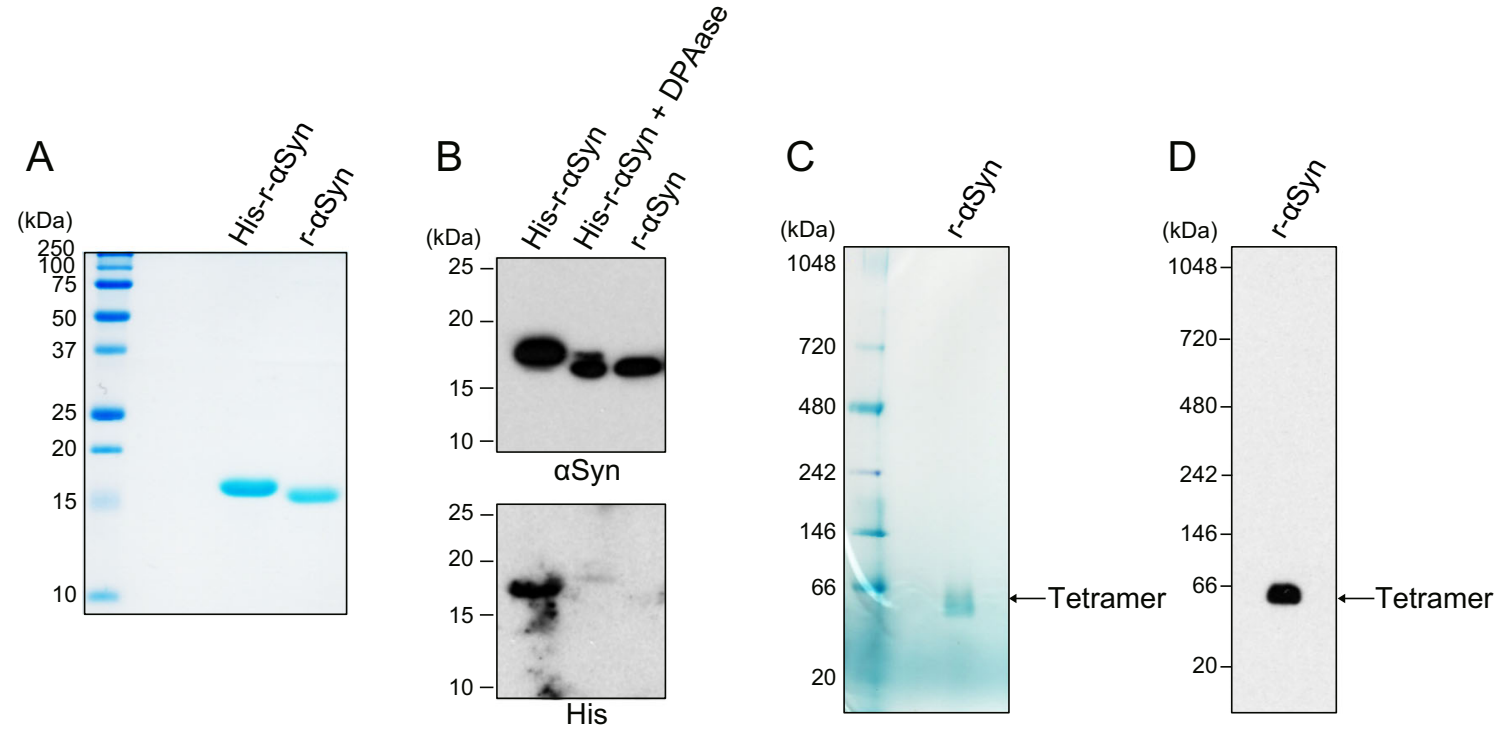

E

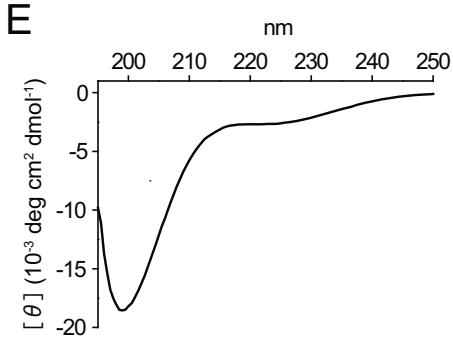

$\mathrm{F}$

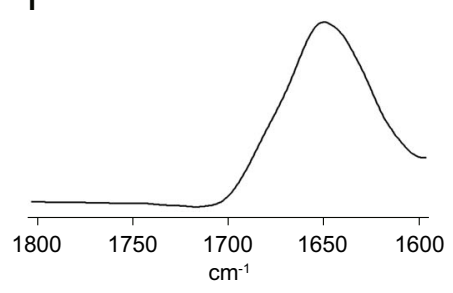

Fig. 1 Purification and structural analysis of recombinant $\alpha$-synuclein. a, b The purified His-tagged recombinant human $\alpha \operatorname{Syn}(H i s-r-\alpha S y n)$, His-r$\alpha$ Syn after treatment with DAPase (His-r- $\alpha$ Syn + DAPase $)$, and the product after removal of His-r- $\alpha$ Syn and DAPase $(r-\alpha S y n)$ were examined by Coomassie brilliant blue $(C B B)$-stained SDS-PAGE and western blotting analysis with polyclonal anti- $\alpha$ Syn antibody D119 and anti-His antibody. a CBB-stained SDS-PAGE analysis showed that the size of His-r- $\alpha$ Syn was shifted from 18 to $17 \mathrm{kDa}$ after treatment with DAPase, and a single major band of the protein was observed after removal of His-r- $\alpha$ Syn and DAPase. b A similar shift in molecular size of His-r- $\alpha$ Syn was also observed by immunoblotting using polyclonal anti- $\alpha$ Syn antibody D119. The purified $r-\alpha$ Syn was confirmed not to be detected by immunoblotting using anti-His-tag antibody. Molecular mass markers are indicated in kilodaltons $(k D a)$ on the left side of each panel. c, d $\mathrm{r}-\alpha$ Syn was loaded onto $4 \%-16 \%$ Bis-Tris native PAGE gels (Invitrogen) and separated by BN-PAGE. c CBB-stained BN-PAGE and $\mathbf{d}$ western blotting analysis with polyclonal anti- $\alpha$ Syn antibody D119 revealed a native $\alpha$ Syn band at approximately $60 \mathrm{kDa}$, suggesting a tetramer (theoretical mass of monomer $=14,460.1 \mathrm{Da}$ ). $\mathbf{e}$ Circular dichroism $(C D)$ spectrum of $\mathrm{r}-\alpha$ Syn showed a minimum mean residue ellipticity at $199 \mathrm{~nm}$, characteristic of disordered protein. f Fourier transform infrared spectroscopy (FTIR) spectrum of $r-\alpha$ Syn. The FTIR spectrum showed a prominent band at $1650 \mathrm{~cm}^{-1}$ assigned to a disordered structure 
products were incubated with Ni-NTA resin at room temperature for $30 \mathrm{~min}$ and loaded onto a gravity flow column to remove the uncleaved His-tagged protein. The protein without His-tag was present in the flow-through fraction. The glutamine residue was converted in the presence of Qcyclase to pyroglutamate, which acts as a stop point for DAPase digestion. The pyroglutamate was eliminated through the action of $1.25 \mathrm{U}$ of pyroglutamyl aminopeptidase (pGAPase, Cat. No. 34342; Qiagen) at $4{ }^{\circ} \mathrm{C}$ overnight, and then the products were loaded onto a gravity flow column after incubation with $\mathrm{Ni}$ NTA resin at room temperature for $30 \mathrm{~min}$. Human $\alpha$ Syn was obtained in the flow-through fraction. DAPase, Qcyclase, and pGAPase carry a His-tag at their C-termini and were therefore removed using Ni-NTA resin. The final protein was dialyzed against $10 \mathrm{mM}$ sodium phosphate buffer ( $\mathrm{pH}$ 7.0) in cellulose dialysis tubing at $4{ }^{\circ} \mathrm{C}$ overnight and filtered with a $0.2-\mu \mathrm{m}$ syringe filter (Cat. No. SLLGH25; Millipore). The purity of the protein samples was $\geq 99.9 \%$, as estimated by SDS-PAGE and immunoblotting, and analysis by nondenaturing Blue Native PAGE (BN-PAGE) revealed a native $\alpha$ Syn band at approximately $60 \mathrm{kDa}$ (Fig. 1c, d), suggesting a tetramer (theoretical mass of monomer $=14,460.1 \mathrm{Da})$. Previous studies have shown that soluble $\mathrm{r}-\alpha$ Syn forms a stable tetramer [21], and endogenous $\alpha$ Syn from normal tissues occurs physiologically as a folded tetramer that resists aggregation [22]. The analysis by circular dichroism (CD) and Fourier transform infrared spectroscopy (FTIR) showed the disordered conformation of $r-\alpha \operatorname{Syn}$ (Fig. 1e, f). After purification, aliquots of the proteins were stored at $-80{ }^{\circ} \mathrm{C}$ until use.

\section{RT-QUIC Experiments}

We prepared reaction mixtures in 96-well, optical, blackbottomed plates with a non-treated surface (Cat. No. 265301; Nunc) in a final total volume of $100 \mu \mathrm{l}$. The surface material was polystyrene. To avoid contamination, we prepared all materials inside a biological safety cabinet and used aerosol-resistant tips. The final concentrations of reaction buffer components were $50 \mathrm{mM}$ HEPES ( $\mathrm{pH}$ 7.5) and $10 \mu \mathrm{M}$ Thioflavin $\mathrm{T}$ (ThT). The concentration of $\mathrm{r}-\alpha \mathrm{Syn}$ was $100-150 \mu \mathrm{g} / \mathrm{ml}$, and only freshly thawed $\mathrm{r}-\alpha$ Syn was used. Brain tissues (frontal cortex region) were homogenized at $10 \%(w / v)$ in ice-cold PBS supplemented with a protease inhibitor mixture (Cat. No. 11836170001; Roche) using a multi-bead shocker. After centrifugation at $2000 \times g$ for 2 min, supernatants were collected and frozen at $-80{ }^{\circ} \mathrm{C}$ until use. Brain homogenate was diluted with PBS prior to the reactions. $r-\alpha$ Syn, suspended in $95 \mu$ l of reaction buffer, was loaded into each well of a 96-well plate and mixed with $5 \mu \mathrm{l}$ of brain homogenate. The 96-well plate was covered with sealing tape (Cat. No. 236366; Nunc) and incubated at $40{ }^{\circ} \mathrm{C}$ in a plate reader (Infinite M200 fluorescence plate reader; TECAN) with intermittent shaking, consisting of $40 \mathrm{~s}$ of circular shaking at the highest speed (432 rpm), no shaking for $20 \mathrm{~s}$, and then a 2 -min pause to measure the fluorescence. The fluorescence intensity on the bottom of the plate was read every $10 \mathrm{~min}$ to monitor the kinetics of amyloid formation using monochromators with excitation and emission wavelengths of 440 and $485 \mathrm{~nm}$, respectively. Six replicates of each diluted brain homogenate sample were measured for $96 \mathrm{~h}$. Each diluted sample of insoluble aggregates and oligomers of $\mathrm{r}-\alpha \mathrm{Syn}$ was assayed in three to four and three to six replicates for $72 \mathrm{~h}$, respectively. The maximal fluorescence intensity in reactions with $5 \times 10^{-5}$ and $5 \times 10^{-6}$ dilutions of non-DLB cases and without seed was $97.3 \pm 6.7$ (means \pm standard deviation) arbitrary units in total 90 wells within 96 h. In addition, none of the reactions reached a fluorescence intensity $>120$ arbitrary units. Therefore, we designated positive reactions as those with fluorescence intensity $>120$ arbitrary units and calculated the seeding dose giving a positive reaction in $50 \%$ of replicate reactions $\left(\mathrm{SD}_{50}\right)$ using the SpearmanKärber method as described previously [23]. We observed slight variations in the optimal $r-\alpha$ Syn concentration (100 $150 \mu \mathrm{g} / \mathrm{ml}$ ) between the lots of $\mathrm{r}-\alpha \mathrm{Syn}$, but the final sensitivity for DN-DLB case \#1, which was used as the standard for calculating sensitivity, was approximately the same $\left(\mathrm{SD}_{50}\right.$ values of $10^{7}-10^{8} / \mathrm{g}$ brain).

\section{Preparation of Insoluble Aggregates and Oligomers of r- $\alpha$ Syn for RT-QUIC Analysis}

Wild-type or S129A $r-\alpha$ Syn $(400 \mu \mathrm{g} / \mathrm{ml})$ was incubated at $37{ }^{\circ} \mathrm{C}$ without agitation in the presence or absence of $4 \mathrm{U} / \mu \mathrm{l}$ of casein kinase 2 (CK2, Cat. No; P6010L, New England Biolabs Inc) or $200 \mu \mathrm{M}$ ATP (Cat. No. A2383-1G; SigmaAldrich) in reaction buffer (20 mM Tris- $\mathrm{HCl}, \mathrm{pH} 7.5,50 \mathrm{mM}$ $\mathrm{KCl}$ and $10 \mathrm{mM} \mathrm{MgCl}_{2}$ ). To obtain insoluble aggregates and oligomers of $r-\alpha$ Syn, the reactions were stopped after 264 and $5 \mathrm{~h}$ of incubation, respectively. After 264-h incubation, the mixture of insoluble $r-\alpha$ Syn aggregates was diluted in $50 \mathrm{mM}$ HEPES buffer ( $\mathrm{pH}$ 7.5) to a final concentration of $125 \mu \mathrm{g} / \mathrm{ml} \mathrm{r}-\alpha$ Syn and stored at $-80{ }^{\circ} \mathrm{C}$ until use. After 5-h incubation, the mixture of $r-\alpha$ Syn oligomers was diluted in $50 \mathrm{mM}$ HEPES buffer ( $\mathrm{pH} 7.5$ ) containing $10 \mu \mathrm{M}$ ThT to a final concentration of $125 \mu \mathrm{g} / \mathrm{ml} \mathrm{r}-\alpha$ Syn and immediately subjected to RT-QUIC. The RT-QUIC products were stored at $-80{ }^{\circ} \mathrm{C}$ until use.

\section{Western Blotting}

Brain tissue (frontal cortex region) was lysed with Tritondeoxycholate (DOC) lysis buffer (50 mM Tris- $\mathrm{HCl}, \mathrm{pH} 7.5$, containing $150 \mathrm{mM} \mathrm{NaCl}, 0.5 \%$ Triton X-100, 0.5\% sodium deoxycholate, $2 \mathrm{mM}$ EDTA, and protease inhibitors) for $30 \mathrm{~min}$ at $4{ }^{\circ} \mathrm{C}$. After $2 \mathrm{~min}$ of centrifugation at $2000 \times \mathrm{g}$, the supernatant was collected, and its total protein 
concentration was measured using a bicinchoninic acid (BCA) protein assay kit (Cat. No. 23227; Pierce). Samples were boiled for $5 \mathrm{~min}$ at $95{ }^{\circ} \mathrm{C}$ with sodium dodecyl sulfate (SDS) loading buffer $(62.5 \mathrm{mM}$ Tris-HCl, $\mathrm{pH} 6.8$, containing $5 \%$ 2-mercaptoethanol, 2\% SDS, 5\% sucrose, and $0.005 \%$ bromophenol blue) and subjected to SDS-polyacrylamide gel electrophoresis (SDS-PAGE). The proteins were transferred onto an Immobilon-P membrane (Cat. No. IPVH304F0; Millipore) in transfer buffer containing 15\% methanol at $300 \mathrm{~mA}$ for $2 \mathrm{~h}$; the membrane was blocked with $5 \%$ nonfat dry milk in TBST $(10 \mathrm{mM}$ Tris- $\mathrm{HCl}, \mathrm{pH} 7.8$, $100 \mathrm{mM} \mathrm{NaCl}, 0.1 \%$ Tween 20) for $2 \mathrm{~h}$ at $4{ }^{\circ} \mathrm{C}$ and reacted with antibodies against $\alpha$ Syn (D119, Cat. No. BS3429; Bioworld Technology, Inc., RRID: AB_1662955, 1:2500), Ser129-phosphorylated $\alpha$ Syn (EP1536Y, Cat. No. ab51253; Abcam, RRID: AB_869973, 1:2500), Ser87-phosphorylated $\alpha$ Syn (Cat. No. sc-19893-R; Santa Cruz Biotechnology, RRID: AB_2192817, 1:500) or His-tag (Cat. No. 34670; Qiagen, RRID: AB_2571551, 1:1000). Immunoreactive bands were visualized by HRP-conjugated goat anti-rabbit IgG antibody (Cat. No. 111-035-003; Jackson ImmunoResearch Labs, RRID: AB_2313567) or goat antimouse IgG antibody (Cat. No. 115-035-003; Jackson ImmunoResearch Labs, RRID: AB_10015289), using an enhanced chemiluminescence system (GE Healthcare Life Sciences).

\section{Transmission Electron Microscopy}

Negative staining was performed on carbon supporting film grids, which were glow-discharged before staining. Aliquots of $5 \mu \mathrm{l}$ of the samples were adsorbed onto the grids, and the residual solution was absorbed with filter paper. The grids were stained with $5 \mu \mathrm{l}$ of freshly filtered stain (2\% uranyl acetate). Once dry, the samples were viewed with a transmission electron microscope (JEM-1400PLUS; JEOL).

\section{CD}

Circular dichroism (CD) spectra were measured with a JASCO J-820 spectropolarimeter (JASCO) using a quartz cell with a 1-mm path length. The CD spectrum was obtained by averaging four scans in the wavelength range of 195 $250 \mathrm{~nm}$. $\mathrm{r}-\alpha$ Syn was dissolved in $20 \mathrm{mM}$ sodium phosphate (pH 6.5) and $150 \mathrm{mM} \mathrm{NaCl}$ buffer. The concentration of $\mathrm{r}-\alpha$ Syn was $300 \mu \mathrm{g} / \mathrm{ml}$.

\section{FTIR}

Fourier transform infrared spectroscopy (FTIR) spectra were measured with a Bruker Tensor 27 FTIR instrument (Bruker Optics) equipped with a mercuric cadmium telluride (MCT) detector cooled with liquid nitrogen. Aliquots of $20 \mu \mathrm{l}$ of the sample were loaded into a BioATRcell II attenuated total reflectance-type reflectance unit. A total of 128 scans at 4$\mathrm{cm}^{-1}$ resolution were collected for each sample under constant purging with nitrogen, corrected for water vapor, and the background spectra of the buffer were subtracted.

\section{Histopathology and Immunohistochemical Staining}

The brain tissues were fixed in $20 \%$ neutral buffered formalin, and $8-\mu \mathrm{m}$ paraffin sections were prepared on glass slides with a microtome. After deparaffinization and rehydration, the tissue sections were subjected to staining with hematoxylin and eosin, and immunohistochemical staining using anti-Ser129phosphorylated $\alpha$ Syn antibody (Cat. No. 015-25191; Wako, RRID: AB_2537218, 1:3000). To enhance the immunogenicity, the sections were prepared by heating for $40 \mathrm{~min}$ at $98^{\circ} \mathrm{C}$ prior to incubation with primary antibody. Primary antibody binding was detected by the labeled streptavidin-biotin method (DAKO). Peroxidase-conjugated streptavidin was visualized with 3'3-diaminobenzidine (Cat. No. 7411-49-6; Wako) as the chromogen. Immunostained sections were lightly counterstained with Mayer's hematoxylin.

\section{Statistical Analysis}

The values of maximal fluorescence intensity and lag phase, the time required to reach a fluorescence intensity $>120$ arbitrary units, in reactions with brain homogenate within $96 \mathrm{~h}$, or in reactions with oligomers of $r-\alpha$ Syn within $72 \mathrm{~h}$ on RTQUIC were extracted, and then compared between dilutions including controls (reactions without seed or with mock sample) in each individual case or sample. In addition, the values in reactions with oligomers of $\mathrm{r}-\alpha \mathrm{Syn}$ were compared between samples at the same dilutions. Data for maximal fluorescence intensities were analyzed by one-way ANOVA, followed by the Tukey-Kramer test. The data for lag phase were subjected to log-rank and Tukey-Kramer tests. $P<0.05$ or $P<0.01$ was taken to indicate statistical significance.

\section{Results}

\section{Ser129-Phosphorylated $\alpha$-Synuclein Is Abundant in the Brain from Dementia with Lewy Bodies}

First, we confirmed the presence of LB and $\alpha$ Syn phosphorylated at Ser129 (pSer129- $\alpha$ Syn) in the brains of DLB patients (Fig. 2a). Both LB and pSer129- $\alpha$ Syn were observed in sections of the substantia nigra and the cortex of the frontal lobe obtained at autopsy from two patients with diffuse neocortical DLB (DN-DLB) and one patient with limbic DLB (Li-DLB). In contrast, histochemical analyses revealed no pathological abnormalities in the brain of a non-DLB case. 

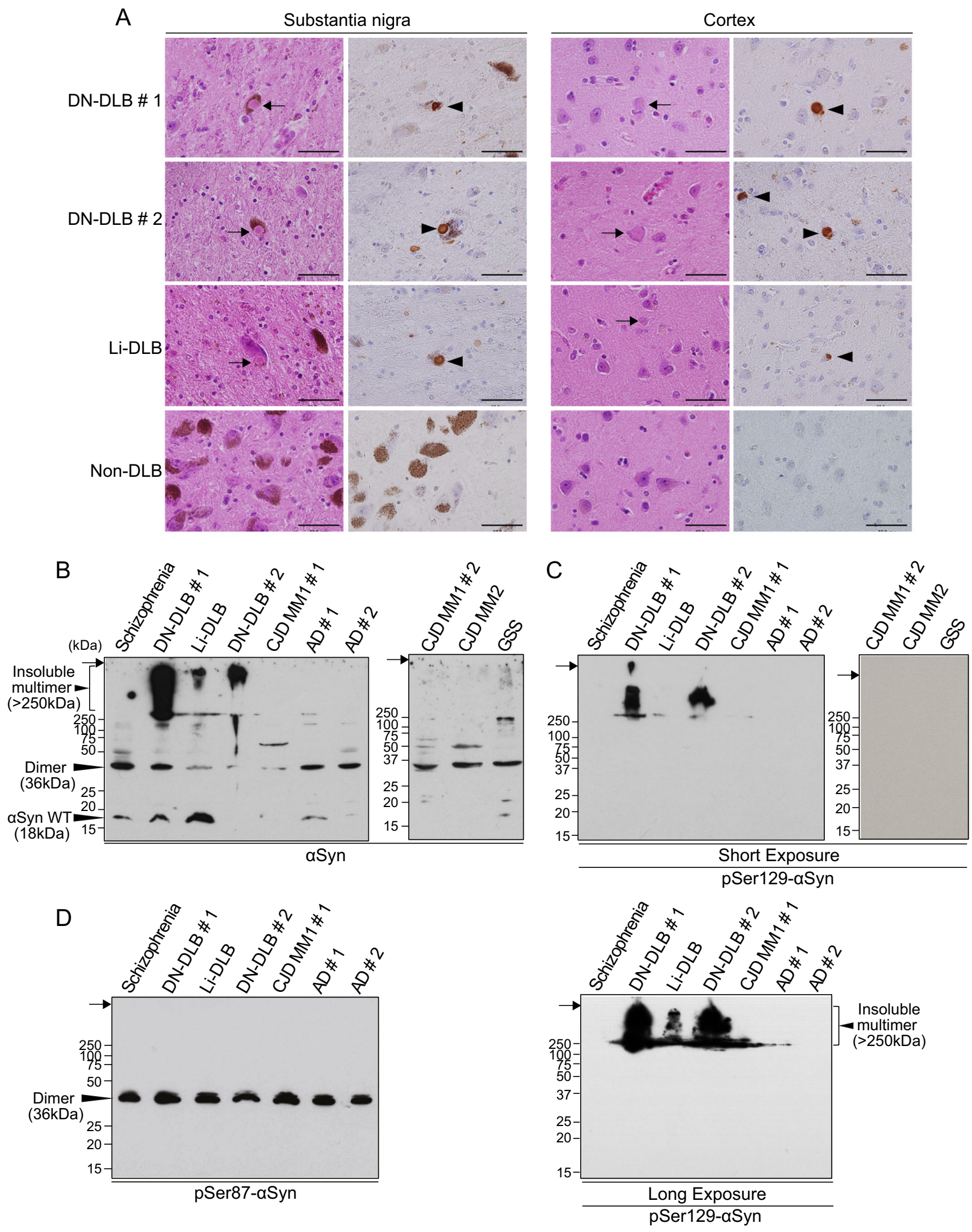
4 Fig. 2 Characterization of $\alpha$-synuclein from human brains by histochemical and western blotting analysis. a Hematoxylin and eosin staining and immunohistochemical staining using an antibody against phosphorylated $\alpha$ Syn in the substantia nigra and the cortex of the frontal lobe from two patients with DN-DLB (cases \#1 and \#2), a patient with Li-DLB, and non-DLB case (breast cancer patient). The arrows and arrowheads show LB and pSer129- $\alpha$ Syn, respectively. Scale bar, $50 \mathrm{~mm}$. b, c, d The samples of BH from DN-DLB (cases \#1 and \#2), Li-DLB, schizophrenia, Alzheimer's disease $(A D)$ (cases \#1 and \#2), sporadic Creutzfeldt-Jakob disease ( $s C J D$ ) type 1 ( $s C J D M M 1$ ) (case \#1 and \#2), sCJD type 2 (sCJD MM2), and GerstmannSträussler-Scheinker syndrome (GSS) patients were immunoblotted with b polyclonal anti- $\alpha$ Syn antibody D119, c monoclonal antipSer129- $\alpha$ Syn antibody, and d polyclonal anti-Ser87-phosphorylated $\alpha$ Syn antibody. In the immunoblotting analysis for pSer129- $\alpha$ Syn presented in $\mathbf{c}$, the samples were detected at a short exposure time of $30 \mathrm{~s}$ (upper panel) and long exposure time of $2 \mathrm{~min}$ (lower panel). Molecular mass markers are indicated in kilodaltons $(k D a)$ on the left side of each panel. The arrows indicate the top of the stacking gel

Consistent with a previous report [24], cells containing melanin granules in the substantia nigra were typically lost in patients with DN-DLB and Li-DLB, but not non-DLB, presumably due to neurodegeneration.

We next examined the biochemical properties of $\alpha$ Syn in brain homogenates $(\mathrm{BH})$ from DLB patients by western blotting (Fig. 2b). Immunoblotting analysis of samples from all patients showed either a native $\alpha$ Syn band at $18 \mathrm{kDa}$, the dimeric form at $36 \mathrm{kDa}$, or both. The two cases of DN-DLB and one case of Li-DLB contained markedly bulky multimeric $\alpha$ Syn, which was in the mass range of $>250 \mathrm{kDa}$. The insoluble multimer was more abundant in DN-DLB than Li-DLB, indicating that $\alpha$ Syn multimer formation is critical to the disease progression. This band was not observed in non-DLB cases. All cases showed bands of various sizes in the mass range of around $50-250 \mathrm{kDa}$. These bands were probably due to the oligomeric and/or ubiquitinated $\alpha$ Syn, and did not differ significantly between cases. The antibody against pSer129- $\alpha$ Syn detected an apparent band at high molecular mass (> $250 \mathrm{kDa}$ ) in two cases of DN-DLB (Fig. 2c). A band of the same size was observed in one case of Li-DLB, but the intensity of immunoreactivity was much lower than in DNDLB. In contrast, non-DLB cases showed no immunoreactivity to the antibody against $\mathrm{pSer} 129-\alpha$ Syn. These observations indicated the presence of an insoluble multimer of $\alpha$ Syn $>$ $250 \mathrm{kDa}$ in the DLB brain that was highly phosphorylated at Ser129. Furthermore, we estimated the levels of pSer129- $\alpha$ Syn in the brains of DLB cases by quantitative dot blot immunoassay following Phos-tag SDS-PAGE (Supplementary Fig. S1). The levels of pSer129- $\alpha$ Syn in DN-DLB cases \#1 and \#2 were $13.5 \pm 0.4$ and $3.7 \pm$ $0.2 \mathrm{mg} / \mathrm{g}$ brain, respectively, and the level of Li-DLB was
$0.06 \pm 0.02 \mathrm{mg} / \mathrm{g}$ brain. The proportion of $\mathrm{pSer} 129-\alpha \mathrm{Syn}$ to total $\alpha$ Syn in DN-DLB was higher than that in Li-DLB: DN-DLB case \#1 $(57.9 \% \pm 1.5 \%)$, DN-DLB \#2 $(22.6 \% \pm$ $1.2 \%)$, and Li-DLB $(6.1 \% \pm 1.7 \%)$. These results suggest that pSer129- $\alpha$ Syn is relevant to disease progression. A previous study indicated that the level of $\alpha$ Syn phosphorylated on Ser87 is also increased in the LBD brain [25]. However, the antibody against Ser87-phosphorylated $\alpha$ Syn detected a dimeric band of $36 \mathrm{kDa}$, and there were no significant differences in levels of the Ser87-phosphorylated form between cases (Fig. 2d).

\section{Conversion of the Soluble Form of Recombinant Human $\alpha$-Synuclein into Amyloid Fibrils by RT-QUIC}

We next examined whether $r-\alpha$ Syn fibril formation could be induced in RT-QUIC by monitoring the levels of ThT fluorescence when $\mathrm{BH}$ from DLB patients was added to the reactions (Fig. 3a, b). When $5 \times 10^{-5}$ and $5 \times 10^{-6}$ dilutions of BH from two cases of DN-DLB were added, all reactions showed positive ThT fluorescence within $96 \mathrm{~h}$. For the Li-DLB case, two of six reactions with a dilution of $5 \times 10^{-5}$ showed positive responses. We also examined the effects of a dilution of $5 \times 10^{-4}$ for Li-DLB, but no increase in fluorescence was observed (data not shown). The negative reaction was probably due to high levels of a variety of components in $\mathrm{BH}$ that inhibit $r-\alpha$ Syn fibril formation in the reactions. In contrast, unseeded controls and all reactions with dilutions of $5 \times 10^{-5}$ and $5 \times 10^{-6}$ schizophrenia $\mathrm{BH}$ produced no response over $96 \mathrm{~h}$. The maximal fluorescence intensity was significantly higher in reactions with dilutions of $5 \times 10^{-5}$ and $5 \times 10^{-6}$ of DN-DLB case \#1, dilutions ranging from $5 \times 10^{-5}$ to $5 \times 10^{-7}$ of DN-DLB case \#2, and a dilution of $5 \times 10^{-5}$ of Li-DLB compared to unseeded controls (Fig. 3b). The lag phase was significantly shorter in reactions with dilutions of $5 \times 10^{-5}$ from two cases of DN-DLB compared to unseeded controls (Fig. 3b). The values of $\mathrm{SD}_{50} / \mathrm{g}$ brain of DN-DLB case \#1 and \#2 were $10^{7.8}$ and $10^{7.3}$, respectively. Although we could not calculate the seeding dose of Li-DLB accurately, the value was estimated to be less than 5.1 (log $\mathrm{SD}_{50} / \mathrm{g}$ brain) based on the assumption that the dilution of $5 \times 10^{-4}$ showed $100 \%$ positivity. The specific detection of DLB was not observed in reactions with recombinant human prion protein as a substrate or protein-free reactions (Supplementary Fig. S2), indicating that DLB demonstrates the ability to seed on $r-\alpha$ Syn but not on other proteins. To further assess the influence of other protein misfolding and degenerative diseases on RT-QUIC, we applied BH from Alzheimer's disease (AD), sporadic Creutzfeldt-Jakob disease (sCJD) type 1 and type 2, and GSS patients to the assay. 
A

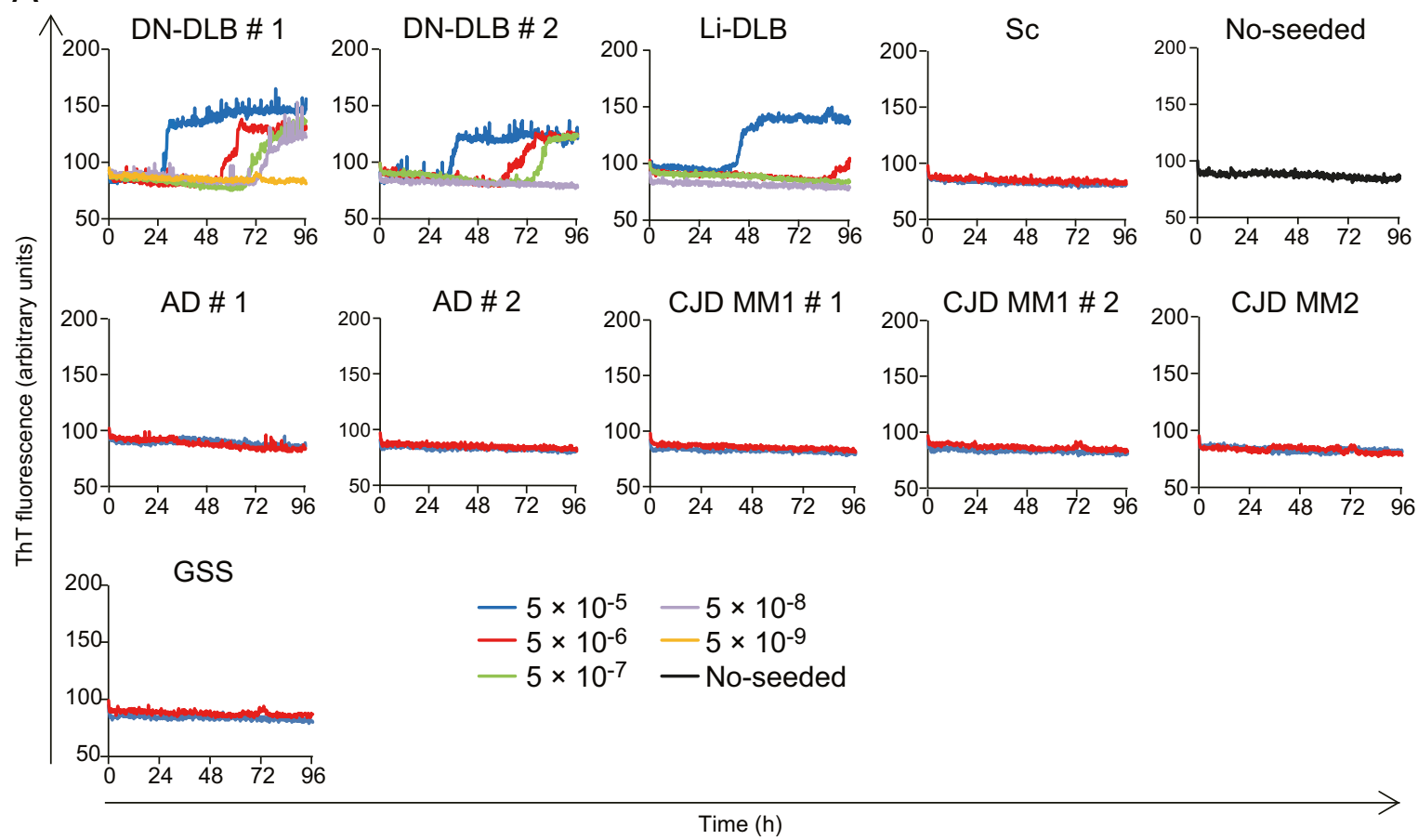

B
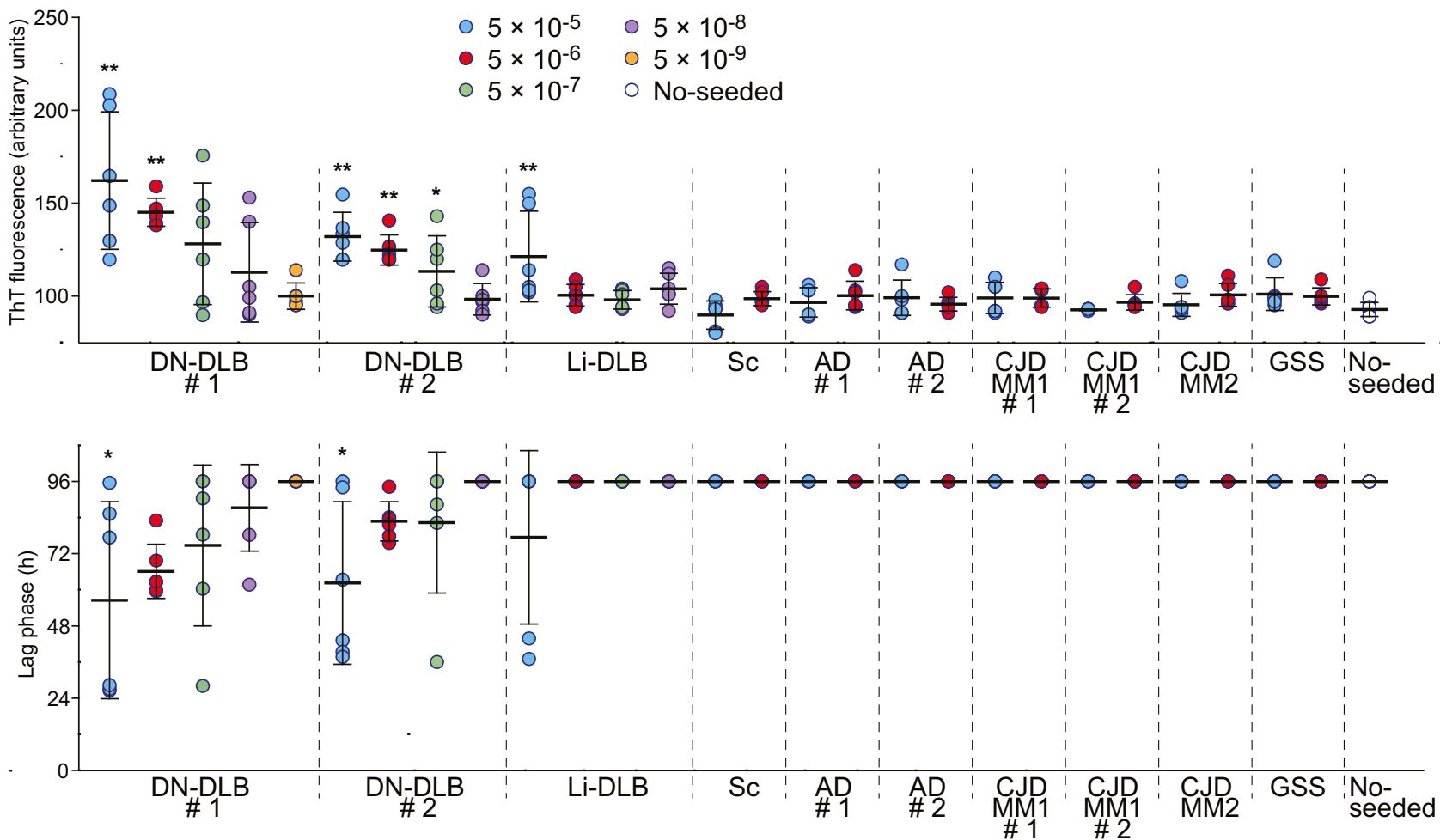

Fig. 3 Fibril formation of $r-\alpha$ Syn in RT-QUIC reactions. a RT-QUIC was performed using human $\mathrm{r}-\alpha \mathrm{Syn}$ with the indicated dilutions of $\mathrm{BH}$ from DN-DLB (cases \#1 and \#2), Li-DLB, schizophrenia ( $S c$ ), AD (cases \#1 and \#2), sCJD MM1 (cases \#1 and \#2), sCJD MM2, and GSS patients, or without seed (no-seeded). The colored curves represent the kinetics of ThT fluorescence average of all six replicate wells. See also Supplementary Fig. S3. b The values of maximal fluorescence intensities and lag phase obtained in individual samples after 96-h reaction are plotted in the upper and lower graphs, respectively. Lag phase was defined as the time required to reach a fluorescence intensity $>120$ arbitrary units. The horizontal bars indicate means \pm standard deviation. The data for maximal fluorescence intensities were analyzed by one-way ANOVA, followed by the Tukey-Kramer test. Analysis of the data for lag phase was performed by the log-rank and Tukey-Kramer tests. ${ }^{*} P<0.01$ (compared with no-seeded) $* P<0.05$ (compared with no-seeded) 
In $\mathrm{AD}, \mathrm{SCJD}$ (type 1 and type 2 ), and GSS, all reactions with dilutions of $5 \times 10^{-5}$ and $5 \times 10^{-6}$ gave negative responses within $96 \mathrm{~h}$ (Fig. 3a, b). These observations indicated that RTQUIC induces the formation of $r-\alpha$ Syn fibrils only in the presence of $\mathrm{BH}$ from DLB cases, and that the seeding activity of DN-DLB is higher than that of Li-DLB. These findings suggest that $r-\alpha$ Syn can be converted into amyloid fibrils through a prion-like mechanism.

To further confirm the reliability of RT-QUIC, we analyzed $\mathrm{BH}$ samples from another four patients with DN-DLB (Fig. 4b, c). Similar to DN-DLB cases \#1 and \#2, immunoblotting analysis of samples from all patients showed an insoluble multimer of $\alpha$ Syn $>250 \mathrm{kDa}$. The pSer129- $\alpha$ Syn was detected only in the multimer size (Fig. $4 \mathrm{a}$ ). When $5 \times 10^{-6}$ and $5 \times 10^{-7}$ dilutions of these cases were added, all of the RTQUIC reactions showed positive. Meanwhile, not all reactions with a dilution of $5 \times 10^{-5}$ were positive, likely due to the presence of endogenous inhibitors of RT-QUIC reaction in $\mathrm{BH}$. The maximal fluorescence intensity was significantly higher in reactions with dilutions from $5 \times 10^{-6}$ and $5 \times 10^{-7}$ in case \#3, ranging from $5 \times 10^{-6}$ to $5 \times 10^{-8}$ in cases $\# 4$, and \#6, and from $5 \times 10^{-6}$ to $5 \times 10^{-9}$ in case \#5 compared to unseeded controls (Fig. 4c). The lag phase was significantly shorter in reactions with dilutions ranging from $5 \times 10^{-6}$ to $5 \times 10^{-8}$ of cases \#3 and \#4 and from $5 \times 10^{-6}$ to $5 \times 10^{-9}$ of cases \#5 and \#6 compared to unseeded controls (Fig. 4c). The values of $\mathrm{SD}_{50} / \mathrm{g}$ brain were as follows: $10^{8.6}$ (case \#3), $10^{9.3}$ (case \#4), $10^{9.8}$ (case \#5), and $10^{9.5}$ (case \#6). Thus, we were able to detect seeding activity of the brains from other DNDLB patients. As shown in Fig. 3a, b, some of the reactions with DN-DLB \#1 and 2 showed positive fluorescence around $24 \mathrm{~h}$ in contrast to the reactions with DN-DLB \#3, 4, 5, and 6. Moreover, we observed differences in the shape of the fluorescence curve between both assays. We used different lots of $\mathrm{r}-\alpha$ Syn for each assay, because the amounts of the lots were not sufficient. A preliminary study using different lots showed that the fluorescence curves were not necessarily similar in reactions with DN-DLB \#1, and there are likely differences in fibril-forming properties, such as the rise time, the intensity of fluorescence, and the shape of the fluorescence curve, between lots of $r-\alpha$ Syn.

\section{Insoluble Aggregates of $\alpha$-Synuclein Had Little or No Seeding Activity}

We next examined whether Ser129 phosphorylation is crucial for $\alpha$ Syn fibril formation through a prion-like mechanism using r- $\alpha$ Syn phosphorylated at Ser129 (pSer129-r- $\alpha$ Syn). WT r- $\alpha$ Syn was phosphorylated at Ser129 by incubation only in the presence of both casein kinase 2 (CK2) and ATP, whereas the S129A mutant was not phosphorylated under the same conditions (Fig. 5a). Similar to the case with DLB BH, the insoluble pSer129-r- $\alpha$ Syn was observed only in the mass range of $>250-\mathrm{kDa}$ after $72-$ and 264-h incubation. Meanwhile, the insoluble aggregates of nonphosphorylated $\mathrm{r}-\alpha$ Syn also converged on the mass range of $>250 \mathrm{kDa}$ after 264-h incubation. Although there were no significant differences in increased levels of ThT fluorescence between WT $\mathrm{r}-\alpha \mathrm{Syn}$ incubated with CK2 in the absence $\left(\mathrm{WT}^{\mathrm{CK} 2}\right.$ ) or presence $\left(\mathrm{WT}^{\mathrm{CK} 2+\mathrm{ATP}}\right)$ of ATP and S129A r- $\alpha$ Syn incubated with CK2 and ATP (S129A ${ }^{\text {CK2+ATP }}$ ) (Fig. 5b), the aggregate formation of pSer129-r- $\alpha$ Syn was induced more efficiently than that of nonphosphorylated $\mathrm{r}-\alpha$ Syn after $72-\mathrm{h}$ incubation (Fig. 5a). These results suggest that Ser129 phosphorylation accelerates polymerization of $r-\alpha$ Syn. Consistent with a previous report [26], a 13-kDa band with molecular weight lower than full-length $r-\alpha$ Syn was observed in all samples after 72-h incubation, indicating that $r-\alpha$ Syn aggregate formation is mediated by the truncation and/or degradation of $r-\alpha$ Syn. FTIR spectra of WT (WT-264 h) and mutant r- $\alpha$ Syn (S129A-264 h) incubated with CK2 and ATP for $264 \mathrm{~h}$ showed shifts to slightly lower wave numbers compared with before incubation (WT-0 h and S129A-0 h), indicating modestly increased $\beta$-sheet content (1630 to $1610 \mathrm{~cm}^{-1}$ ) (Fig. $5 \mathrm{c}$ ). As shown in Supplementary Table 1 , the $\beta$-sheet contents of WT-264 h $(36.6 \%)$ and S129A-264 $\mathrm{h}(45.2 \%)$ were higher than those of WT-0 h (24.3\%) and S129A-0 h (27.9\%). There was little difference in the infrared spectra between WT and mutant $r-\alpha$ Syn before or after incubation (Fig. $5 c$ ). Transmission electron microscopy (TEM) analysis indicated that WT-264 $\mathrm{h}$ and S129A-264 h consisted exclusively of amorphous aggregates (Fig. 5d). We next examined whether $r-\alpha$ Syn could be newly converted into amyloid fibrils in the presence of amorphous $\mathrm{r}-\alpha$ Syn aggregates in the RT-QUIC. Unexpectedly, all of the reactions with WT-264 h or S129A-264 h at dilutions of $2 \times 10^{-2}$ and $2 \times 10^{-4}$ yielded negative results in the RTQUIC assay (Fig. 5e). Thus, the insoluble aggregates of $\mathrm{r}-\alpha$ Syn had no prion-like seeding activity regardless of whether they were phosphorylated on Ser129 or nonphosphorylated.

\section{Oligomer-Like Forms of $\alpha$-Synuclein Cause Prion-Like Propagation}

RT-QUIC using WT r- $\alpha$ Syn resulted in a more rapid increase and higher levels of fluorescence intensity in the reactions with CK2 in the presence of ATP (WT ${ }^{\mathrm{CK} 2+\mathrm{ATP}}$ ) than in its absence $\left(\mathrm{WT}^{\mathrm{CK} 2}\right)$, whereas there were no significant differences in ThT binding kinetics of S129A r- $\alpha$ Syn between the two conditions (S129A ${ }^{\mathrm{CK} 2}$ and S129A ${ }^{\mathrm{CK} 2+\mathrm{ATP}}$ ) (Fig. 6a). The antibody against $\mathrm{pSer} 129-\alpha$ Syn detected a predominant band at $16 \mathrm{kDa}$ and a weak band in the mass range of $>250 \mathrm{kDa}$ only in $\mathrm{WT}^{\mathrm{CK} 2+\mathrm{ATP}}$ (Fig. 6b). These results suggest that Ser129 phosphorylation accelerates fibril formation of $r-\alpha$ Syn in the RT-QUIC. Unlike the insoluble aggregates generated without shaking, as shown in Fig. 5a, all of the reactions predominantly showed a monomeric $\alpha$ Syn band at 
A
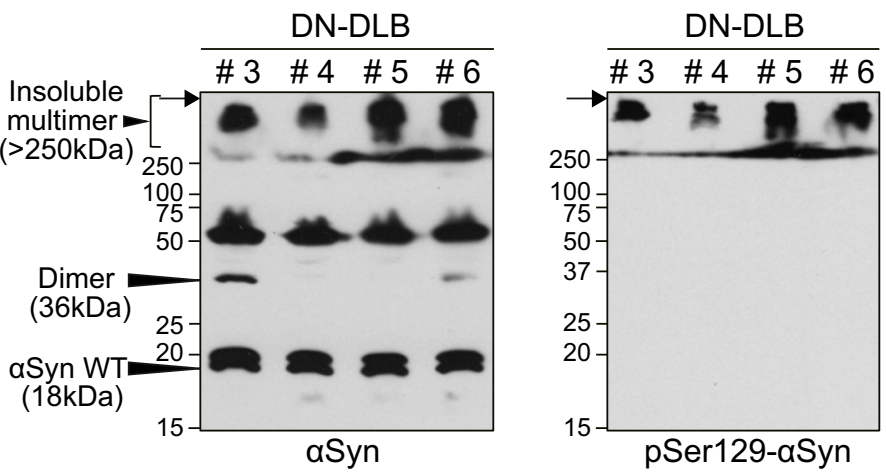

B

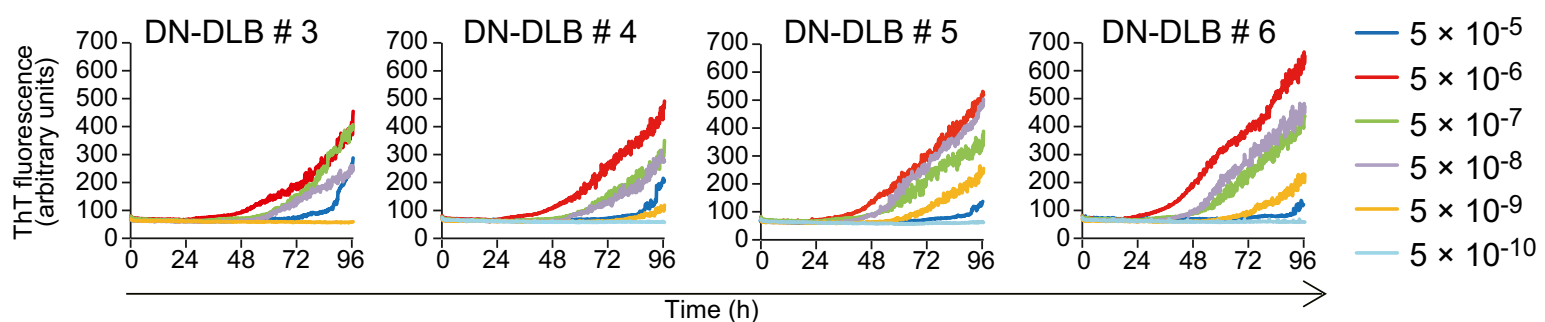

C
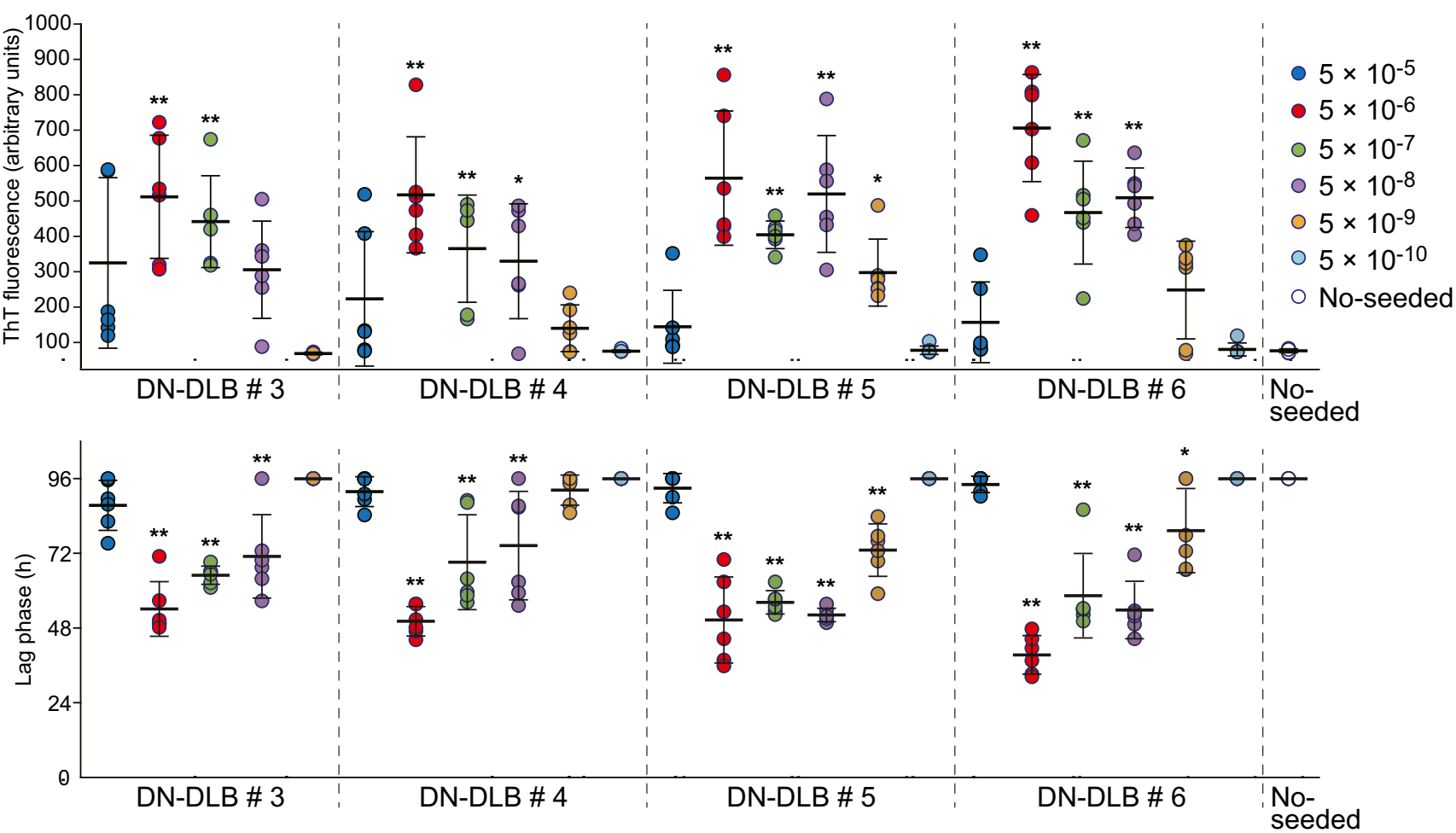

Fig. 4 Western blotting and RT-QUIC analysis of another four DN-DLB brains. a The samples of BH from DN-DLB patients (cases \#3, \#4, \#5 and \#6) were immunoblotted with polyclonal anti- $\alpha$ Syn antibody D119 and monoclonal anti-pSer129- $\alpha$ Syn antibody. Molecular mass markers are indicated in kilodaltons $(k D a)$ on the left side of each panel. The arrows indicate the top of the stacking gel. b RT-QUIC was performed using human $\mathrm{r}-\alpha$ Syn with the indicated dilutions of BH from DN-DLB. The colored curves represent the kinetics of ThT fluorescence average of all six replicate wells. See also Supplementary Fig. S4. c The values of

$16 \mathrm{kDa}$. In addition, the polymer larger than $250 \mathrm{kDa}$ was barely detectable in $\mathrm{WT}^{\mathrm{CK} 2+\mathrm{ATP}}$ and $\mathrm{WT}^{\mathrm{CK} 2}$. The difference maximal fluorescence intensities and lag phase obtained in individual samples after 96-h reaction are plotted in the upper and lower graphs, respectively. Lag phase was defined as the time required to reach a fluorescence intensity $>120$ arbitrary units. The horizontal bars indicate means \pm standard deviation. The data for maximal fluorescence intensities were analyzed by one-way ANOVA, followed by the Tukey-Kramer test. Analysis of the data for lag phase was performed by the log-rank and Tukey-Kramer tests. $* * P<0.01$ (compared with no-seeded); ${ }^{*} P<0.05$ (compared with no-seeded)

in aggregation size of $r-\alpha$ Syn from Fig. 5a was probably due to shaking, which may cause the fragmentation of fibrils. 

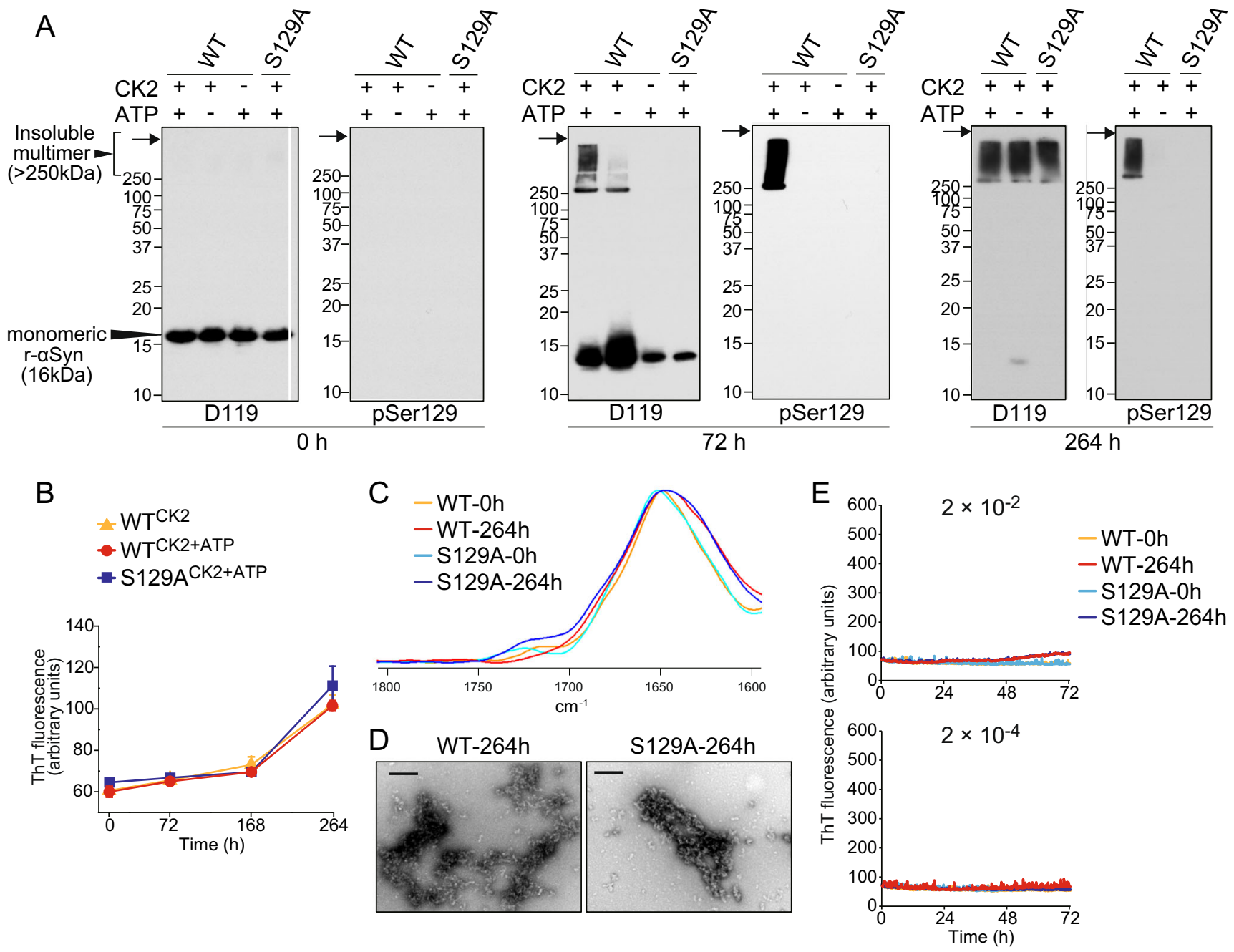

Fig. 5 Seeding activity of insoluble aggregates of $r-\alpha \operatorname{Syn}$ induced by incubation. WT or S129A r- $\alpha$ Syn $(14 \mu \mathrm{g})$ was incubated at $37{ }^{\circ} \mathrm{C}$ in the presence $(+)$ or absence $(-)$ of $140 \mathrm{U}$ of casein kinase 2 (CK2) or $200 \mu \mathrm{M}$ ATP in $35 \mu$ of reaction buffer $(20 \mathrm{mM}$ Tris- $\mathrm{HCl}, \mathrm{pH} 7.5,50 \mathrm{mM} \mathrm{KCl}$, and $10 \mathrm{mM} \mathrm{MgCl}_{2}$ ). a After 0 (left panel), 72 (central panel), or $264 \mathrm{~h}$ (right panel) of incubation, the samples were immunoblotted with polyclonal anti- $\alpha$ Syn antibody D119 and monoclonal anti-pSer129- $\alpha$ Syn antibody. Molecular mass markers are indicated in kilodaltons $(k D a)$ on the left side of each panel. The arrows indicate the top of the stacking gel. b Levels of ThT fluorescence of WT r- $\alpha$ Syn mixed with CK2 in the

absence $\left(\mathrm{WT}^{\mathrm{CK} 2}\right.$ ) or presence (WT ${ }^{\mathrm{CK} 2+\mathrm{ATP}}$ ) of ATP and S129A r- $\alpha \mathrm{Syn}$ mixed with CK2 and ATP (S129A $\left.{ }^{\text {CK2+ATP }}\right)$ were measured after 0, 72, 168, and $264 \mathrm{~h}$ of incubation. Data are expressed as means \pm standard deviation $(n=4)$. $\mathbf{c}$ WT or S129A r- $\alpha$ Syn incubated with CK2 and ATP for 0 (WT$0 \mathrm{~h}$ and S129A-0 h) or $264 \mathrm{~h}$ (WT-264 $\mathrm{h}$ and S129A-264 h) were subjected to FTIR analysis. d Samples were examined by TEM. Bars, $200 \mathrm{~nm}$. e Seeding activity of WT- $0 \mathrm{~h}$, WT-264 h, S129A-0 h, and S129A-264 h samples was evaluated at dilutions of $2 \times 10^{-2}$ and $2 \times 10^{-4}$ by RTQUIC. The colored curves represent the kinetics of ThT fluorescence averaged over three or four replicate wells

FTIR analysis showed that there was little difference in the prominent band at $1650 \mathrm{~cm}^{-1}$ assigned to the disordered

structure among all reactions (Fig. 6c). TEM analysis of $\mathrm{WT}^{\mathrm{CK} 2+\mathrm{ATP}}$ and $\mathrm{S} 129 \mathrm{~A}^{\mathrm{CK} 2+\mathrm{ATP}}$ revealed oligomer-like

Table 1 Assignments and relative proportions of secondary conformations of WT or S129A r- $\alpha$ Syn incubated with CK2 and ATP for 0 (WT- $0 \mathrm{~h}$ and S129A-0 h) or $264 \mathrm{~h}$ (WT-264 h and S129A-264 h). The secondary structure content was determined by curve-fitting analysis of amide I components

\begin{tabular}{|c|c|c|c|c|c|c|c|c|}
\hline \multicolumn{2}{|l|}{ WT-0 h } & \multicolumn{2}{|l|}{ WT-264 h } & \multicolumn{2}{|l|}{ S129A-0 h } & \multicolumn{2}{|l|}{ S129A-264 h } & \multirow[b]{2}{*}{ Assignment } \\
\hline Peak $\left(\mathrm{cm}^{-1}\right)$ & Area $(\%)$ & Peak $\left(\mathrm{cm}^{-1}\right)$ & Area (\%) & Peak $\left(\mathrm{cm}^{-1}\right)$ & Area (\%) & Peak $\left(\mathrm{cm}^{-1}\right)$ & Area (\%) & \\
\hline 1626 & 24.3 & 1625 & 36.6 & 1626 & 27.9 & 1629 & 45.2 & $\beta$-Sheet \\
\hline 1642 & 30.2 & 1645 & 26.3 & 1643 & 29.0 & 1646 & 23.4 & Disordered \\
\hline 1654 & 34.5 & 1658 & 29.8 & 1656 & 32.3 & 1659 & 23.4 & $\alpha$-Helix \\
\hline 1670 & 11.0 & 1678 & 7.3 & 1672 & 10.8 & 1681 & 8.0 & $\beta$-Turn \\
\hline
\end{tabular}


A

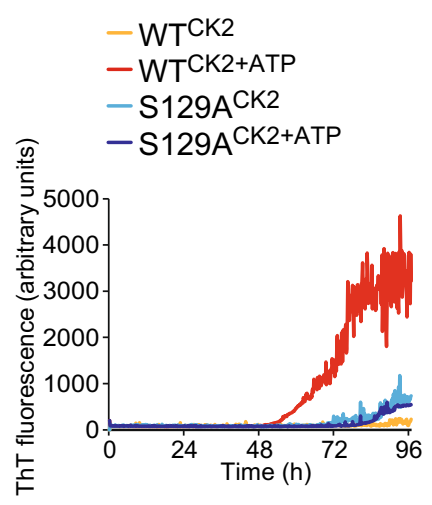

B

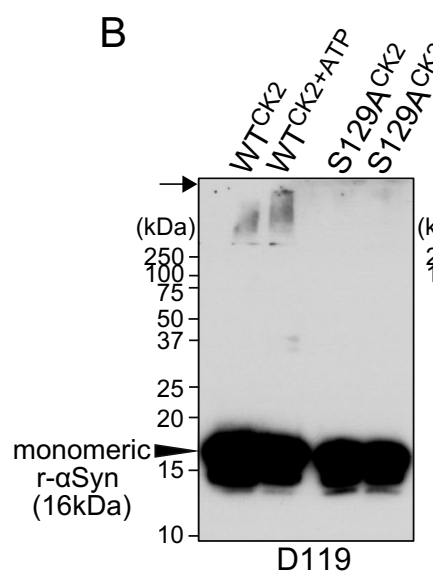

D119

\section{8}<smiles>[131IH]</smiles>

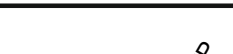

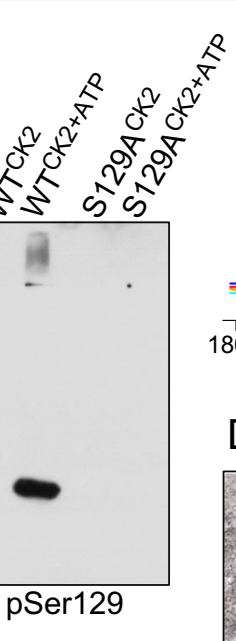

否

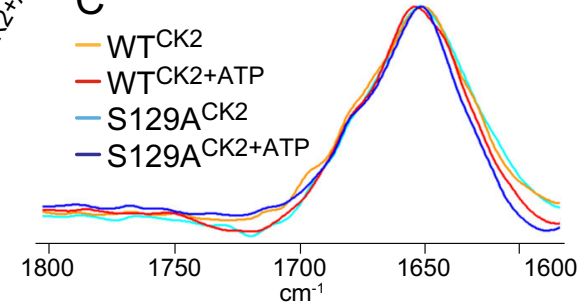
E $\quad-$ WTCK2+ATP
$-W T C K 2$
$-\mathrm{S} 129 \mathrm{~A}^{\mathrm{CK} 2+\mathrm{ATP}}$

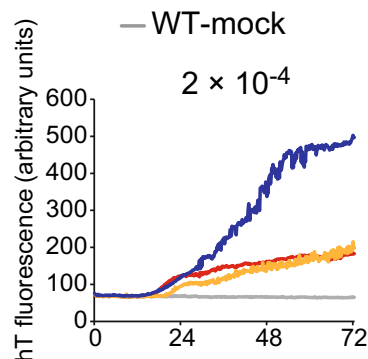
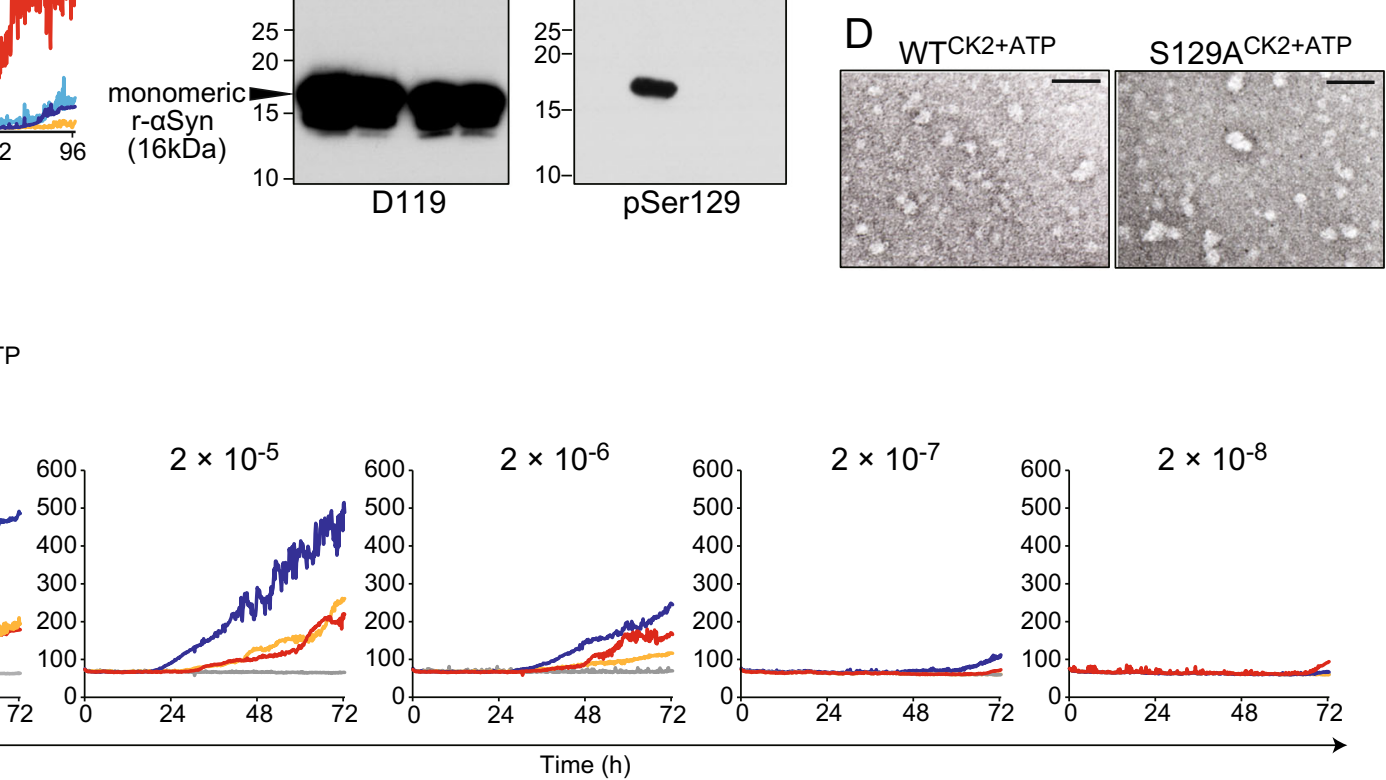

\section{$\mathrm{F}$}
○ $2 \times 10^{-4}$
- $2 \times 10^{-5}$
- $2 \times 10^{-7}$
○ $2 \times 10^{-6}$
○ $2 \times 10^{-8}$
o WT-mock $\left(2 \times 10^{-4}\right)$
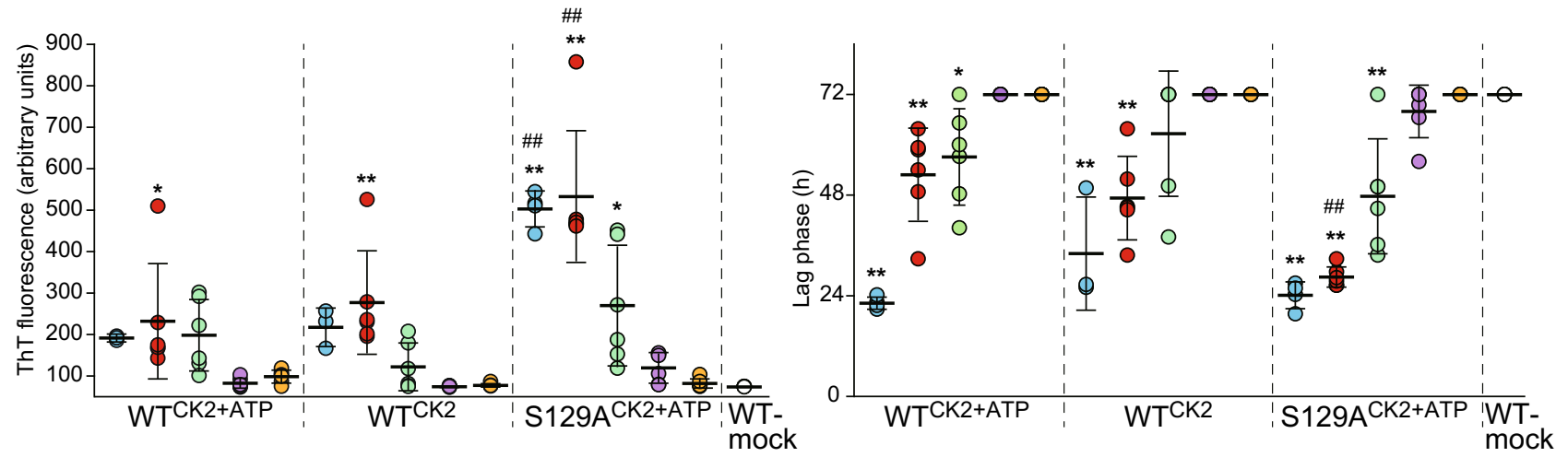

Fig. 6 Seeding activity of $r-\alpha$ Syn oligomers generated by RT-QUIC. a Fibril formation of WT or S129A r- $\alpha$ Syn $(12.5 \mu \mathrm{g})$ was induced in the presence of only $125 \mathrm{U}$ of CK2 (WT ${ }^{\mathrm{CK} 2}$ and $\mathrm{S} 129 \mathrm{~A}^{\mathrm{CK} 2}$ ) or both $125 \mathrm{U}$ of CK2 and $200 \mu \mathrm{M}$ ATP (WT ${ }^{\text {CK2+ATP }}$ and S129A ${ }^{\text {CK2+ATP }}$ ) by RT-QUIC without seed. The colored curves represent the kinetics of ThT fluorescence averaged over triplicate wells. b RT-QUIC samples $\left(\mathrm{WT}^{\mathrm{CK} 2}, \mathrm{WT}^{\mathrm{CK} 2+\mathrm{ATP}}, \mathrm{S} 129 \mathrm{~A}^{\mathrm{CK} 2}\right.$, and $\mathrm{S} 129 \mathrm{~A}^{\mathrm{CK} 2+\mathrm{ATP}}$ ) were immunoblotted with polyclonal anti- $\alpha$ Syn antibody D119 and monoclonal anti-pSer129- $\alpha$ Syn antibody. Molecular mass markers are indicated in kilodaltons $(k D a)$ on the left side of each panel. The arrows indicate the top of the stacking gel. c RT-QUIC samples (WT ${ }^{\mathrm{CK} 2}$, $\mathrm{WT}^{\mathrm{CK} 2+\mathrm{ATP}}$, S129A $\mathrm{A}^{\mathrm{CK} 2}$, and S129A $\mathrm{CK}^{\mathrm{C} 2+\mathrm{ATP}}$ ) were subjected to FTIR analysis. d Samples were examined by TEM. Bars, $50 \mathrm{~nm}$. e Seeding activity of RT-QUIC samples (WT ${ }^{\mathrm{CK} 2}$, WT ${ }^{\mathrm{CK} 2+\mathrm{ATP}}, \mathrm{S} 129 \mathrm{~A}^{\mathrm{CK} 2}$, and
$\mathrm{S} 129 \mathrm{~A}^{\mathrm{CK} 2+\mathrm{ATP}}$ ) was evaluated at dilutions from $2 \times 10^{-4}$ to $2 \times 10^{-8}$ by subsequent testing by RT-QUIC. The colored curves represent the kinetics of ThT fluorescence averaged over replicate wells $(n=3-6)$. f The values of maximal fluorescence intensities and lag phase obtained in individual samples after 72-h reaction are plotted on the left and right graphs, respectively. Lag phase was defined as the time required to reach a fluorescence intensity $>120$ arbitrary units. The horizontal bars indicate means \pm standard deviation. The data for maximal fluorescence intensities were analyzed by one-way ANOVA, followed by the Tukey-Kramer test. Analysis of the data for lag phase was performed by the log-rank and the Tukey-Kramer tests. $* * P<0.01$ (compared with $2 \times 10^{-4}$ dilution of WT-mock); $* P<0.05$ (compared with $2 \times 10^{-4}$ dilution of WT-mock); \#\#P<0.01 (compared with the same dilution of $\mathrm{WT}^{\mathrm{CK} 2+\mathrm{ATP}}$ ) 
granular forms of $r-\alpha \operatorname{Syn}$ (Fig. 6d). To examine whether these oligomer-like species show seeding activity, we performed a second passage of the RT-QUIC samples (Fig. 6e, f). The dilutions of $2 \times 10^{-4}$ and $2 \times 10^{-5}$ of $\mathrm{WT}^{\mathrm{CK} 2+\mathrm{ATP}}$, $\mathrm{WT}^{\mathrm{CK} 2}$, and S129A ${ }^{\text {CK2+ATP }}$ showed $100 \%$ positivity in all of the reactions. The phosphorylated oligomer-like species yielded an $\mathrm{SD}_{50}$ value of $10^{4.9} / \mu \mathrm{gr}-\alpha \operatorname{Syn}\left(\mathrm{WT}^{\mathrm{CK} 2+\mathrm{ATP}}\right.$ ), and the nonphosphorylated oligomer-like species showed $\mathrm{SD}_{50}$ values of $10^{4.4} / \mu \mathrm{g} r-\alpha \operatorname{Syn}\left(\mathrm{WT}^{\mathrm{CK} 2}\right)$ and $10^{5.4} / \mu \mathrm{g} \mathrm{r}-\alpha \operatorname{Syn}\left(\mathrm{S} 129 \mathrm{~A}^{\mathrm{CK} 2+}\right.$ ATP). In contrast, we observed no increase in fluorescence in any reactions with dilutions ranging from $2 \times 10^{-4}$ to $2 \times 10^{-8}$ of a mock sample (WT-mock) that contained the same components as $\mathrm{WT}^{\mathrm{CK} 2+\mathrm{ATP}}$, which was prepared without shaking immediately before the assay. The maximal fluorescence intensity was significantly higher in reactions with $\mathrm{WT}^{\mathrm{CK} 2+\mathrm{ATP}}$ and $\mathrm{WT}^{\mathrm{CK} 2}$ at a dilution of $2 \times 10^{-5}$ and dilutions ranging from $2 \times 10^{-4}$ to $2 \times 10^{-6}$ of S129A ${ }^{\mathrm{CK} 2+\mathrm{ATP}}$ compared to WT-mock (Fig. 6f). The lag phase was significantly shorter in reactions with dilutions ranging from $2 \times 10^{-4}$ to $2 \times 10^{-6}$ of WT ${ }^{\mathrm{CK} 2+\mathrm{ATP}}$ and $\mathrm{S} 129 \mathrm{~A}^{\mathrm{CK} 2+\mathrm{ATP}}$ and $2 \times 10^{-4}$ and $2 \times 10^{-5}$ dilutions of $\mathrm{WT}^{\mathrm{CK} 2}$ compared to WT-mock (Fig. 6f). Our results indicated that oligomer-like species of $r-\alpha$ Syn could display seeding activity with or without phosphorylation at Ser129.

\section{Discussion}

The results of this study demonstrated that the formation of $r-\alpha$ Syn fibrils is induced by RT-QUIC using soluble $r-\alpha$ Syn only in the presence of BH from patients with DLB (Fig. 3a, b). The sodium dodecyl sulfate (SDS)-insoluble aggregates larger than $250 \mathrm{kDa}$ detected only in DLB cases were specifically phosphorylated at Ser129 (Fig. 2c), and therefore we postulated that the insoluble aggregates of pSer129- $\alpha$ Syn confer prion-like seeding activity. Unexpectedly, however, the insoluble aggregates of $r-\alpha$ Syn with increased $\beta$-sheet structure had little seeding activity in both phosphorylated and nonphosphorylated states (Fig. 5e). Instead, the pre-fibrillar oligomers of $r-\alpha$ Syn exerted seeding activity both with and without phosphorylation (Fig. 6e, f). Our findings suggest that the soluble oligomeric, but not fully fibrillary, $\alpha$ Syn is a seeding species in vitro. Meanwhile, inoculation of fibrils generated from $r-\alpha$ Syn has been reported to induce prion-like spreading of pathological $\alpha$ Syn in WT mice [17]. The synthetic fibrils were formed with agitation $\left(1000 \mathrm{rpm}\right.$ at $\left.37^{\circ} \mathrm{C}\right)$, and sonicated briefly prior to inoculation into mice, whereas the insoluble aggregates of $r-\alpha$ Syn used in Fig. 5e were generated by incubation at $37{ }^{\circ} \mathrm{C}$ without agitation. The fibril samples obtained using agitation and sonication are likely to contain oligomeric forms of $\mathrm{r}-\alpha \mathrm{Syn}$, because these procedures are known to cause fibril fragmentation, resulting in shorter fibrils $[27,28]$. Moreover, the fibrils may undergo partial proteolytic degradation in vivo. Therefore, the prion-like properties of synthetic $\mathrm{r}-\alpha$ Syn fibrils reported in previous in vivo studies may be causally related to $r-\alpha$ Syn oligomers present in the fibril preparation and/or produced by degradation in vivo. Previous studies have shown that oligomeric species of $r-\alpha$ Syn are internalized by primary neurons and neuronal cell lines and induce endogenous $\alpha$ Syn aggregation $[29,30]$. Furthermore, there is substantial evidence suggesting that oligomeric forms of $\alpha$ Syn are responsible for neuronal cell death and neurodegeneration [31, 32]. In a postmortem study, levels of soluble $\alpha$ Syn oligomers were significantly higher in the brains of patients with DLB than AD and controls, while there were no significant differences in levels of total $\alpha$ Syn between the three groups [33]. These reports support the suggestion that pre-fibrillar $\alpha$ Syn oligomers represent the pathogenic species with prion-like behavior in LBD rather than mature fibrils or amorphous aggregates. A similar approach using monomeric amyloid- $\beta(A \beta)$ indicated that the protein misfolding cyclic amplification (PMCA) assay could detect seeding activity associated with $\mathrm{A} \beta$ oligomers present in CSF from patients with AD [34]. Brain tissue from DNDLB had $\mathrm{SD}_{50}$ values of $10^{3.4}$ and $10^{3.1} / \mu \mathrm{g}$ total $\alpha$ Syn in cases \#1 and \#2, respectively, and $10^{2.1} / \mu \mathrm{g}$ total $\alpha \mathrm{Syn}$ in LiDLB (Fig. 3a, b), while the values of WT r- $\alpha$ Syn oligomers generated by RT-QUIC were $10^{4.4}-10^{4.9} / \mu \mathrm{g} \mathrm{r}-\alpha \mathrm{Syn}$ (Fig. 6e, $\mathrm{f})$. It is unclear whether all of the $r-\alpha$ Syn in RT-QUIC reactions is present in oligomeric forms, but if so $3.2-10 \%$ and $1.6-5.0 \%$ of total $\alpha$ Syn are estimated to be oligomers in the brains of DN-DLB cases \#1 and \#2, respectively, and $0.2-$ $0.5 \%$ in that of Li-DLB. Although the precise role of the LB insoluble aggregates remains unclear, cyto- and neuroprotective roles have been reported in studies using cell lines [35] and Drosophila [4]. The seeding activity of S129A oligomers was higher than that of WT oligomers regardless of phosphorylation state (Fig. 6f), and small amounts of the insoluble aggregates were detected in reactions containing WT oligomers but not S129A oligomers on western blotting analysis (Fig. 6b). These results suggest that the insoluble aggregates confer protection against prion-like propagation of $\alpha$ Syn.

Although there were no significant differences in seeding activity of $r-\alpha$ Syn oligomers between Ser129-phosphorylated and nonphosphorylated forms, we found that the phosphorylation of $r-\alpha$ Syn accelerates self-assembly (Fig. 5a and Fig. 6a) consistent with previous reports [1]. Previous studies showed that an increase in pSer129- $\alpha$ Syn level precedes the appearance of LB in the brains of patients with LBD [36] and is induced by oxidative stress, mitochondrial dysfunction [37], and proteasomal inhibition [38] observed in the LBD pathology. Furthermore, pSer129- $\alpha$ Syn has been reported to have a protective effect against neuronal dysfunction $[5,39]$. These results suggest that $p$ Ser129- $\alpha$ Syn results from the protective mechanism against neuronal dysfunction. The pSer129- $\alpha$ Syn would then promote initiation of self-assembly into prefibrillar oligomers and mature fibrils. 
Consistent with the previous report [20], RT-QUIC allowed the differentiation of DLB from other degenerative disorders, such as $\mathrm{AD}$ and prion diseases, as well as from nondegenerative cases, suggesting that $r-\alpha$ Syn is largely unaffected by the ability of other misfolded proteins, i.e., amyloid- $\beta$, tau, and $\operatorname{PrP}^{\mathrm{Sc}}$, to induce heterologous cross-seeding. Although there have been reports of cross-seeding interaction between $\alpha$ Syn and other misfolded proteins in vitro and in vivo [40], the effect on RT-QUIC reaction appears to be negligible. The discrimination of DLB from other degenerative disorders raises the possibility of application of specific detection using RT-QUIC to differential diagnosis. The brains from patients with DN-DLB had an $\mathrm{SD}_{50}$ value of $10^{7-10} / \mathrm{g}$ brain, and that with Li-DLB was estimated to have an $\mathrm{SD}_{50}$ value of approximately $10^{5.1} / \mathrm{g}$ brain. Thus, it was suggested that RT-QUIC has a higher detection sensitivity for DLB compared with testing for pSer129- $\alpha$ Syn using western blotting or ELISA and provided more precise brain biopsy or autopsy diagnosis. Several studies using ELISA or bead-based flow cytometric assay investigated the levels of total $\alpha$ Syn in the CSF and blood of patients with DLB and other synucleinopathies, but the results have been inconclusive and contradictory [41]. Meanwhile, it was shown that the levels of soluble $\alpha$ Syn oligomers in CSF and blood of DLB and PD are elevated compared with those of $\mathrm{AD}$ and controls in ELISA [42, 43]. These reports support the suggestion that oligomeric forms of $\alpha$ Syn are significant and promising targets for diagnosis of LBD and suggest the diagnostic potential of RT-QUIC through seeding of oligomers from CSF and blood of patients with LBD. Thus, the use of RT-QUIC along with the existing ELISA specific for $\alpha$ Syn oligomers is likely to be particularly advantageous in the differential diagnosis of LBD. The sample size was a relatively small for comparison between the different types of DLB because Li-DLB was examined in only one case in this study. Further replication studies with larger samples are warranted to determine whether RT-QUIC is valuable for discrimination between the different types of LBD, as well as whether this assay can be used with body fluids or other tissues.

In this study, we demonstrated the feasibility of using RTQUIC for laboratory detection of potential seeding species of $\alpha$ Syn. Our data provide further support for the suggestion that the oligomeric forms of $\alpha \mathrm{Syn}$ are the seeding species that cause prion-like propagation and play a significant role in the pathogenesis of LBD. We believe that this new method represents a robust tool for clinical diagnosis, screening for potential drugs, and advances our understanding of the role of $\alpha$ Syn as a prion-like protein in LBD.

Acknowledgements We thank Dr. Tsuyoshi Mori and Dr. Takayuki Fuse for helpful discussions, and Atsuko Matsuo for technical assistance. This work was supported by a grant-in-aid for challenging exploratory research (grant no. 25640029) from the Ministry of Education, Culture, Sports, Science, and Technology of Japan.

Authors' Contributions K. Sano., R.A., and K. Satoh. designed the entire project. K. Sano and R.A. wrote the manuscript. K. Sano., D.I., and T.N. performed the experiments and analyzed the data. K. Satoh., Y.I., M.Y., and S.M. contributed to the collection of human specimens and provided information about subjects. R.A., K.M., and N.N. supervised and discussed the data.

Compliance with Ethical Standards The study protocol was approved by the Ethics Committee of Nagasaki University Hospital (ID: 10042823) and registered with the University Hospital Medical Information Network (ID: UMIN000003301). Written informed consent for inclusion in the study was given by the families of all patients.

Conflict of Interest The authors declare that they have no conflict of interest.

Open Access This article is distributed under the terms of the Creative Commons Attribution 4.0 International License (http:// creativecommons.org/licenses/by/4.0/), which permits unrestricted use, distribution, and reproduction in any medium, provided you give appropriate credit to the original author(s) and the source, provide a link to the Creative Commons license, and indicate if changes were made.

\section{References}

1. Fujiwara H, Hasegawa M, Dohmae N et al (2002) Alpha-synuclein is phosphorylated in synucleinopathy lesions. Nat Cell Biol 4:160164

2. Smith WW, Margolis RL, Li X et al (2005) Alpha-synuclein phosphorylation enhances eosinophilic cytoplasmic inclusion formation in SH-SY5Y cells. J Neurosci : the official journal of the Society for Neuroscience 25:5544-5552

3. Paleologou KE, Schmid AW, Rospigliosi CC et al (2008) Phosphorylation at Ser-129 but not the phosphomimics S129E/D inhibits the fibrillation of alpha-synuclein. J Biol Chem 283: 16895-16905

4. Chen L, Feany MB (2005) Alpha-synuclein phosphorylation controls neurotoxicity and inclusion formation in a Drosophila model of Parkinson disease. Nat Neurosci 8:657-663

5. Gorbatyuk OS, Li S, Sullivan LF et al (2008) The phosphorylation state of Ser-129 in human alpha-synuclein determines neurodegeneration in a rat model of Parkinson disease. Proc Natl Acad Sci U S A 105:763-768

6. Azeredo da Silveira S, Schneider BL, Cifuentes-Diaz C et al (2009) Phosphorylation does not prompt, nor prevent, the formation of alpha-synuclein toxic species in a rat model of Parkinson's disease. Hum Mol Genet 18:872-887

7. McFarland NR, Fan Z, Xu K et al (2009) Alpha-synuclein S129 phosphorylation mutants do not alter nigrostriatal toxicity in a rat model of Parkinson disease. J Neuropathol Exp Neurol 68:515-524

8. Braak H, Del Tredici K, Rub U et al (2003) Staging of brain pathology related to sporadic Parkinson's disease. Neurobiol Aging 24:197-211

9. Braak H, Rub U, Jansen Steur EN et al (2005) Cognitive status correlates with neuropathologic stage in Parkinson disease. Neurology 64:1404-1410

10. Kordower JH, Chu Y, Hauser RA et al (2008) Lewy body-like pathology in long-term embryonic nigral transplants in Parkinson's disease. Nat Med 14:504-506 
11. Li JY, Englund E, Holton JL et al (2008) Lewy bodies in grafted neurons in subjects with Parkinson's disease suggest host-to-graft disease propagation. Nat Med 14:501-503

12. Luk KC, Kehm VM, Zhang B et al (2012) Intracerebral inoculation of pathological alpha-synuclein initiates a rapidly progressive neurodegenerative alpha-synucleinopathy in mice. J Exp Med 209: 975-986

13. Mougenot AL, Nicot S, Bencsik A et al (2012) Prion-like acceleration of a synucleinopathy in a transgenic mouse model. Neurobiol Aging 33:2225-2228

14. Watts JC, Giles K, Oehler A et al (2013) Transmission of multiple system atrophy prions to transgenic mice. Proc Natl Acad Sci U S A 110:19555-19560

15. Masuda-Suzukake M, Nonaka T, Hosokawa M et al (2013) Prionlike spreading of pathological alpha-synuclein in brain. Brain : a journal of neurology 136:1128-1138

16. Volpicelli-Daley LA, Luk KC, Patel TP et al (2011) Exogenous alpha-synuclein fibrils induce Lewy body pathology leading to synaptic dysfunction and neuron death. Neuron 72:57-71

17. Luk KC, Kehm V, Carroll J et al (2012) Pathological alphasynuclein transmission initiates Parkinson-like neurodegeneration in nontransgenic mice. Science (New York, NY) 338:949-953

18. Atarashi R, Satoh K, Sano K et al (2011) Ultrasensitive human prion detection in cerebrospinal fluid by real-time quaking-induced conversion. Nat Med 17:175-178

19. Sano K, Atarashi R, Ishibashi D et al (2014) Conformational properties of prion strains can be transmitted to recombinant prion protein fibrils in real-time quaking-induced conversion. J Virol 88: 11791-11801

20. Fairfoul G, McGuire LI, Pal S et al (2016) Alpha-synuclein RTQuIC in the CSF of patients with alpha-synucleinopathies. Ann Clin Transl Neurol 3:812-818

21. Wang W, Perovic I, Chittuluru J et al (2011) A soluble alphasynuclein construct forms a dynamic tetramer. Proc Natl Acad Sci U S A 108:17797-17802

22. Bartels T, Choi JG, Selkoe DJ (2011) Alpha-synuclein occurs physiologically as a helically folded tetramer that resists aggregation. Nature 477:107-110

23. Wilham JM, Orru CD, Bessen RA et al (2010) Rapid end-point quantitation of prion seeding activity with sensitivity comparable to bioassays. PLoS Pathog 6:e1001217

24. Thannickal TC, Lai YY, Siegel JM (2007) Hypocretin (orexin) cell loss in Parkinson's disease. Brain : a journal of neurology 130: 1586-1595

25. Paleologou KE, Oueslati A, Shakked G et al (2010) Phosphorylation at $\mathrm{S} 87$ is enhanced in synucleinopathies, inhibits alpha-synuclein oligomerization, and influences synucleinmembrane interactions. J Neurosci : the official journal of the Society for Neuroscience 30:3184-3198

26. Vlad C, Lindner K, Karreman C et al (2011) Autoproteolytic fragments are intermediates in the oligomerization/aggregation of the Parkinson's disease protein alpha-synuclein as revealed by ion mobility mass spectrometry. Chembiochem : a European journal of chemical biology 12:2740-2744
27. Chatani E, Lee YH, Yagi H et al (2009) Ultrasonication-dependent production and breakdown lead to minimum-sized amyloid fibrils. Proc Natl Acad Sci U S A 106:11119-11124

28. Xue WF, Hellewell AL, Gosal WS et al (2009) Fibril fragmentation enhances amyloid cytotoxicity. J Biol Chem 284:34272-34282

29. Danzer KM, Krebs SK, Wolff M et al (2009) Seeding induced by alpha-synuclein oligomers provides evidence for spreading of alpha-synuclein pathology. J Neurochem 111:192-203

30. Danzer KM, Haasen D, Karow AR et al (2007) Different species of alpha-synuclein oligomers induce calcium influx and seeding. $\mathrm{J}$ Neurosci : the official journal of the Society for Neuroscience 27: 9220-9232

31. Brown DR (2010) Oligomeric alpha-synuclein and its role in neuronal death. IUBMB life 62:334-339

32. Vekrellis K, Xilouri M, Emmanouilidou E et al (2011) Pathological roles of alpha-synuclein in neurological disorders. Lancet Neurol 10:1015-1025

33. Paleologou KE, Kragh CL, Mann DM et al (2009) Detection of elevated levels of soluble alpha-synuclein oligomers in postmortem brain extracts from patients with dementia with Lewy bodies. Brain : a journal of neurology 132:1093-1101

34. Salvadores N, Shahnawaz M, Scarpini E et al (2014) Detection of misfolded Abeta oligomers for sensitive biochemical diagnosis of Alzheimer's disease. Cell Rep 7:261-268

35. Tanaka M, Kim YM, Lee G et al (2004) Aggresomes formed by alpha-synuclein and synphilin-1 are cytoprotective. J Biol Chem 279:4625-4631

36. Lue LF, Walker DG, Adler CH et al (2012) Biochemical increase in phosphorylated alpha-synuclein precedes histopathology of Lewytype synucleinopathies. Brain Pathol (Zurich, Switzerland) 22:745756

37. Perfeito R, Lazaro DF, Outeiro TF et al (2014) Linking alphasynuclein phosphorylation to reactive oxygen species formation and mitochondrial dysfunction in SH-SY5Y cells. Mol Cell Neurosci 62:51-59

38. Chau KY, Ching HL, Schapira AH et al (2009) Relationship between alpha synuclein phosphorylation, proteasomal inhibition and cell death: relevance to Parkinson's disease pathogenesis. J Neurochem 110:1005-1013

39. Kuwahara T, Tonegawa R, Ito G et al (2012) Phosphorylation of alpha-synuclein protein at Ser-129 reduces neuronal dysfunction by lowering its membrane binding property in Caenorhabditis elegans. J Biol Chem 287:7098-7109

40. Morales R, Moreno-Gonzalez I, Soto C (2013) Cross-seeding of misfolded proteins: implications for etiology and pathogenesis of protein misfolding diseases. PLoS Pathog 9:e1003537

41. Kasuga K, Nishizawa M, Ikeuchi T (2012) Alpha-synuclein as CSF and blood biomarker of dementia with Lewy bodies. Int J Alzheimers Dis 2012:437025

42. Tokuda T, Qureshi MM, Ardah MT et al (2010) Detection of elevated levels of alpha-synuclein oligomers in CSF from patients with Parkinson disease. Neurology 75:1766-1772

43. Hansson O, Hall S, Ohrfelt A et al (2014) Levels of cerebrospinal fluid alpha-synuclein oligomers are increased in Parkinson's disease with dementia and dementia with Lewy bodies compared to Alzheimer's disease. Alzheimers Res Ther 6:25 